\title{
Propagation of a finite-amplitude potential vorticity front along the wall of a stratified fluid
}

\author{
By MELVIN E. STER ${ }^{1}$ AND KARL R. HELFRICH \\ ${ }^{1}$ Department of Oceanography, Florida State University, Tallahassee, FL 32306, USA \\ stern@ocean.fsu.edu \\ ${ }^{2}$ Department of Physical Oceanography, Woods Hole Oceanographic Institution, \\ Woods Hole, MA 02543, USA \\ khelfrich@whoi.edu
}

(Received 6 November 2001 and in revised form 2 May 2002)

A similarity solution to the long-wave shallow-water equations is obtained for a density current (reduced gravity $=g^{\prime}$, Coriolis parameter $=f$ ) propagating alongshore $(y=0)$. The potential vorticity $q=f / H_{1}$ is uniform in $-\infty<x \leqslant x_{\text {nose }}(t)$, $0<y \leqslant L(x, t)$, and the nose of this advancing potential vorticity front displaces fluid of greater $q=f / H_{0}$, which is located at $L<y<\infty$. If $L_{0}=L(-\infty, t)$, the nose point with $L\left(x_{\text {nose }}(t), t\right)=0$ moves with velocity $U_{\text {nose }}=\sqrt{g^{\prime} H_{0}} \phi$, where $\phi$ is a function of $H_{1} / H_{0}, f^{2} L_{0}^{2} / g^{\prime} H_{0}$. The assumptions made in the similarity theory are verified by an initial value solution of the complete reduced-gravity shallow-water equations. The latter also reveal the new effect of a Kelvin shock wave colliding with a potential vorticity front, as is confirmed by a laboratory experiment. Also confirmed is the expansion wave structure of the intrusion, but the observed values of $U_{\text {nose }}$ are only in qualitative agreement; the difference is attributed to the the presence of small-scale (non-hydrostatic) turbulence in the laboratory experiment but not in the numerical solutions.

\section{Introduction}

In the classical non-rotating dam-break problem (Stoker 1957) a vertical crosschannel wall (dam) separates water of non-dimensional depth $h=1$ from water of the same density, but greater depth $h=H>1$. Lifting the wall causes a gravity wave to surge downstream $(x>0)$ uniformly at all cross-stream positions $(y)$. In the rotating case (Gill 1976; Stern 1980; Stern, Whitehead \& Hu 1982; Griffiths \& Hopfinger 1983; Griffiths 1986; Herman, Rhines \& Johnson 1989; Tomasson \& Melville 1992; Helfrich, Kuo \& Pratt 1999) lifting the dam also causes a gravity wave; in this case it is a Kelvin wave that propagates downstream on the right-hand wall (looking downstream, with northern hemisphere convention) into the high-potentialvorticity $(q)$ fluid. This Kelvin wave establishes a geostrophic boundary current on the right-hand wall that is fed upstream, ultimately by a boundary current on the left-hand wall (itself initiated by an upstream-propagating Kelvin wave). These two boundary currents are connected by a cross-channel jet in the neighbourhood of the $\operatorname{dam}(x=0)$.

This fundamental problem in the dynamics of rotating stratified fluids has been solved in the linear limit (infinitesimal $H-1$ ) by Gill (1976) who found that the crosschannel jet and the material surface separating the high- and low-potential-vorticity 
fluids (the potential vorticity front) remains at the initial location of the dam. The numerical problem is complicated at finite amplitude because the turbulence that develops at the leading edge of the steepening Kelvin wave requires an ad hoc 'shock joining' condition (see Helfrich et al. 1999 and the references therein). Another theoretical difficulty lies in connecting the cross-channel jet with the downstream 'geostrophic jet' after the former encounters a stagnation point on the wall. For small, but finite, $H-1$ some aspects of the dynamics of the potential vorticity front have been isolated in a weakly nonlinear theory (Herman et al. 1989). Their Rossby number expansion of the shallow-water equations removes direct consideration of the fast Kelvin wave and gives the more tractable quasi-geostrophic equations. Numerical solutions of these equations show that nonlinearity leads to the downstream advection of the potential vorticity front along both channel walls. The intrusion speed is greatest on the right-hand side, where a non-steepening wedge ('rarefaction wave') forms for a sufficiently wide channel. If the channel is very narrow, the intrusion steepens into a shock-like front.

Herman et al. (1989) and Helfrich et al. (1999) used numerical solutions of the shallow-water equations to study the evolution for larger $H-1$ and found qualitative agreement with the weakly nonlinear result for the downstream propagation of a rarefying potential vorticity front along the right-hand wall. Helfrich et al. (1999) found that the intrusion speed increased with increasing $H-1$ and increasing channel width. Importantly, in these nonlinear studies the intrusion nose speed is always less than the speed of the leading nonlinear Kelvin wave. Only in the limit of $H \rightarrow \infty$ does the intrusion speed approach the leading Kelvin wave speed. Both of these studies employed eddy viscous forces which violate potential vorticity conservation; this is generally unrealistic from the point of view of large-scale ocean dynamics, as well as from the point of view of laboratory experiments (Whitehead, Leetma \& Knox 1974; Stern et al. 1982; Griffiths \& Hopfinger 1983) even though (real) wall friction does modify the potential vorticity dynamics.

The dynamics of rotating boundary currents is a fundamental fluid dynamics problem with many geophysical applications. Examples similar to the dam-break problem outlined above include the penetration of coastally trapped disturbances in the marine atmospheric boundary layer along the west coast of the United States (Beardsley et al. 1987; Dorman 1987; Rogerson 1999), and the relaxation of a downwelling ocean front of finite length along the coast after the cessation of the wind. In both situations a thick localized region of fluid adjacent to a boundary is laterally surrounded by a shallower layer with the same density. Relaxation of this non-equilibrium state will produce a Kelvin wave followed by the intrusion of fluid parcels with low potential vorticity along the boundary. The evolution of this intrusion (or alternatively the material surface which defines the potential vorticity front) is the focus of this work. In view of the difficulties associated with a purely numerical approach, and as an important benchmark, it seems desirable to formulate a simpler version of the finite-amplitude dam-break problem. The main goal of the paper is to isolate the 'slow' dynamics of the potential vorticity front from the faster dynamics associated with gravity waves.

In $\S 2$ we obtain a long-wave theory for the evolution of a potential vorticity front in a model which removes the previously mentioned complications of the cross-channel jet and stagnation point; consequently the dammed fluid is now of finite transverse (y) extent (figures 1 and 9). Our theory focuses on the advance of the nose of the potential vorticity front (rather than the faster Kelvin wave), and leads to a rarefaction wave (in agreement with the earlier work) whose intrusion speed $U_{\text {nose }}$ is calculated. 

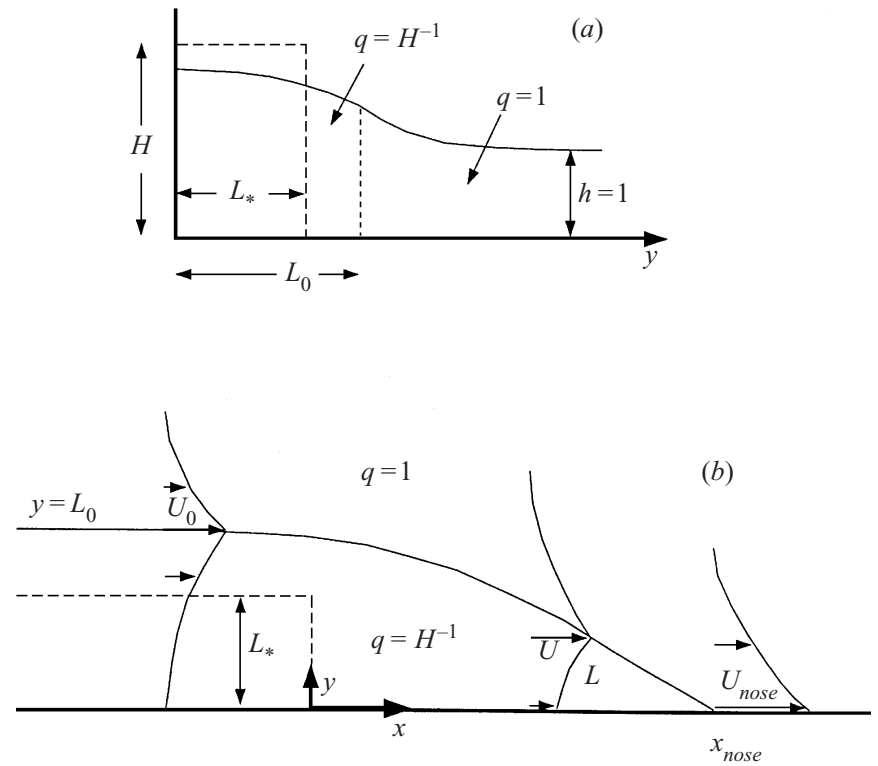

FIGURE 1. Schematic of problem setup: (a) Side view looking downstream at $x \rightarrow-\infty$. $H$ is the initial height and $L_{*}$ is the initial width of the fluid inside the dam; $h(y)$ is the equilibrium thickness after the dam is removed. The dashed line indicated by the equilibrium width $L_{0}$ separates fluid of different potential vorticities. (b) Plan view. Potential vorticity front $L(x, t)$ with $L=0$ at $x_{\text {nose }}$ is sketched along with the $y$-profile of $U$ at various $x$. The leading Kelvin shock (not shown) is at $x>x_{\text {nose }}$.

In $\S 3$ the theory is compared with numerical solutions of the complete shallow-water equations for the initial value dam-break problem (figure 1) in which Poincare and Kelvin waves emerge as well as a potential vorticity front. This is followed in $\S 4$ with results from some simple laboratory experiments and in $\S 5$ by more numerical results which explore aspects of the laboratory experiments which are not present in the original formulation of the theory. The results are summarized and discussed in $\S 6$.

\section{Long-wave theory}

Consider a rotating fluid (figure 1$)$ in $y \geqslant 0$ which contains a semi-infinite $(-\infty<$ $x<0$ ) dam (dashed rectangle), whose non-dimensional width $L_{*}$ is scaled by the radius of deformation $L_{D}=\sqrt{g^{\prime} H_{0}} / f$, where $f$ is the Coriolis parameter, $g^{\prime}$ is the reduced gravity, and $H_{0}$ is the dimensional depth of the fluid outside the dam. The dam contains motionless liquid of non-dimensional depth $h=H>1\left(H=H_{1} / H_{0}\right)$, and outside the dam the fluid with $h=1$ has the same density. This active layer is bounded above by a motionless, infinitely deep layer of lower density leading to a reduced-gravity, or $1 \frac{1}{2}$-layer model. (Note that the active layer could be buoyant and over-ride a deep lower layer with identical results.) When the dam is lifted the fluid at $x=-\infty$ will be displaced outwards $(y>0)$ in an $x$-independent motion which conserves non-dimensional potential vorticity $q=(1+\partial v / \partial x-\partial u / \partial y) h^{-1}$, as in the classic Rossby adjustment problem. Here $u$ and $v$ are the non-dimensional velocities in the $x$ - and $y$-directions, respectively, and have been scaled by $\sqrt{g^{\prime} H_{0}}$. Both $x$ and $y$ are scaled by $L_{D}$. As shown in $\S 2.1$ a steady laminar flow is established at $x=-\infty$ with only a downstream geostrophic component $u$. This upstream equilibrium (Rossby) 
state has an interface at some $y=L(-\infty, t)=L_{0}$ that separates fluid initially behind the dam from the displaced fluid initially lying outside the dam. The equilibrium $L_{0}$ and velocity $u\left(-\infty, L_{0}, t\right) \equiv U(-\infty, t) \equiv U_{0}$ depend only on the governing parameters such as $L_{*}$ and $H$ (see below).

On the other hand, the fluid near $x=0$ at $t=0$ will shoot downstream $(x>0)$ along the wall $(y=0)$ with uniform $q=H^{-1}$ in $0<y<L(x, t)$. All the fluid outside this front $(y>L(x, t))$ has $q=1$, and some of this originates at $x \ll 0$ where it was pushed outwards (and downstream) in the Rossby adjustment process. At some later time the nose of the low- $q$ intrusion is at $x_{\text {nose }}(t)$, where $L\left(x_{\text {nose }}, t\right)=0$ and $u\left(x_{\text {nose }}, t\right)=U_{\text {nose }}$. As previously mentioned, we tentatively assume (and subsequently verify) that the leading edge of the Kelvin wave lies downstream from $x_{\text {nose }}$, and has no effect on the potential vorticity front. We also assume that at the times of interest the bounding interface $L(x, t)$ is a slowly varying function of $x<x_{\text {nose }}(t)$ which smoothly joins the aforementioned laminar upstream state, i.e.

$$
L(x, t) \rightarrow L_{0}, \quad U(x, t) \rightarrow U_{0} \quad(x \rightarrow-\infty)
$$

are the upstream boundary conditions (as obtained in $\S 2.1$ ) for $L(x, t)$.

At some time after the removal of the dam we assume the long-wave equations apply to the downstream $L(x, t)$. If $\delta^{2}=\left(L_{D} / L_{x}\right)^{2} \ll 1$ and $v / u=O(\delta)$, where $L_{x}$ is the length scale in the $x$-direction, the semi-geostrophic equations (e.g. Stern 1986) are

$$
\begin{gathered}
\frac{\partial u}{\partial t}-\left(1-\frac{\partial u}{\partial y}\right) v=-\frac{\partial}{\partial x}\left(h+\frac{u^{2}}{2}\right), \\
u=-\frac{\partial h}{\partial y}, \\
\frac{\partial h}{\partial t}+\frac{\partial(u h)}{\partial x}+\frac{\partial(v h)}{\partial y}=0 .
\end{gathered}
$$

For piecewise-uniform potential vorticity the $y, v$ variables may be eliminated $(\S 2.2)$ to obtain hyperbolic partial differential equations for the interfacial variables $L(x, t)$, $U(x, t)$. We will find two 'simple waves' with characteristic speed $c(L, U)(\mathrm{cf}$. $\$ 2.3$ ), one of which will connect 'properly' with the nose point where $L=0, v=\mathrm{d} L / \mathrm{d} t=0$, $u=U\left(x_{\text {nose }}(t), t\right) \equiv U_{\text {nose }}$. We also assume that the region $x \geqslant x_{\text {nose }}$, where $q=1$, contains the rear part of the Kelvin wave with $v=0, u(x, y, t)=-\partial h / \partial y, u(x, 0, t)=$ $U_{\text {nose }}$. We emphasize that although the foregoing assumptions are physically plausible (Ansatz), they are testable by solving the initial-value shallow-water equations (§3.2). But first $(\$ 2.3)$ we consider the similarity theory for $H=\infty$ (zero potential vorticity inside the dam) because of its relative simplicity, and then $(\S 2.4)$ the finite potential vorticity case is discussed. Following the numerical verification $(\S 3)$, an experimental realization $(\S 4)$ is obtained.

\subsection{Boundary conditions}

As previously stated, after the removal of the dam in figure 1 the fluid at $x=$ $-\infty$ undergoes a rapid Rossby adjustment with $\partial / \partial x=0$. The transverse velocity $v(-\infty, L, t)=\mathrm{d} L / \mathrm{d} t$ displaces the interface from $y=L_{*}$ to a new steady value of $y=L_{0}$, with geostrophic $x$-velocity $u$. Since there is no downstream pressure gradient in this region, and since $v(-\infty, 0, t)=0$, the downstream wall velocity $u(-\infty, 0, t)$ is

$$
u(-\infty, 0)=0 \quad(\text { all } t)
$$


and the temporal integration of $\mathrm{d} u / \mathrm{d} t-v=0$ yields the interfacial velocity

$$
u\left(-\infty, L_{0}\right)=L_{0}-L_{*} .
$$

At all $y$, the geostrophic relation is

$$
u=-\frac{\partial h}{\partial y},
$$

and the piecewise-uniform value of potential vorticity (in the semi-geostrophic approximation) is

$$
q=\frac{1+\partial^{2} h / \partial y^{2}}{h}= \begin{cases}1, & y>L_{0} \\ H^{-1}, & y<L_{0} .\end{cases}
$$

The solution of (7) and (8) for $y>L_{0}$ gives a value of $u=h-1$ which is proportional to $\mathrm{e}^{-y}$. Since $u$ is continuous across the interface, it follows that the steady-state boundary condition for $y \leqslant L_{0}$ is

$$
U\left(L_{0}\right) \equiv u\left(-\infty, L_{0}\right)=h\left(-\infty, L_{0}\right)-1 \text {. }
$$

For $y \leqslant L_{0}$ the solutions to (7) and (8) subject to (5) are

$$
\begin{gathered}
u(-\infty, y)=U\left(L_{0}\right) \frac{\sinh \left(y / H^{1 / 2}\right)}{\sinh \left(L_{0} / H^{1 / 2}\right)}, \\
h-H=-U\left(L_{0}\right) H^{1 / 2} \frac{\cosh \left(y / H^{1 / 2}\right)}{\sinh \left(L_{0} / H^{1 / 2}\right)},
\end{gathered}
$$

and (9) becomes

$$
U\left(L_{0}\right)=\frac{H-1}{1+H^{1 / 2} \operatorname{coth}\left(L_{0} / H^{1 / 2}\right)} .
$$

This important relation will provide the upstream boundary condition for $U(x, t)$ and $L(x, t)$. Although the independent variable for the dam-break problem is $L_{*}$, rather than $L_{0}$, the latter quantity and $U\left(L_{0}\right)$ are implicitly related to $L_{*}$ by $(6)$ and $(10)$, or

$$
U\left(L_{0}\right)=L_{0}-L_{*} .
$$

We will also need a boundary condition at the 'nose point' $x_{\text {nose }}(t)$ where

$$
L\left(x_{\text {nose }}(t), t\right)=0, \quad U_{\text {nose }}=U\left(x_{\text {nose }}(t), t\right)>0 .
$$

As previously mentioned the region $x>x_{\text {nose }}(t)$ is assumed to be the trailing part of the Kelvin wave in which a uniform $u(x, y, t)=U_{\text {nose }} \mathrm{e}^{-y}$ exists. To ensure that $x_{\text {nose }}$ trails the leading edge of the Kelvin disturbance (not shown in figure 1) we will require that the local propagation velocity of the leading Kelvin shock, $\Gamma_{K}$, computed for $x>x_{\text {nose }}$ satisfies

$$
\Gamma_{K}>U_{\text {nose }} .
$$

Fedorov \& Melville (1996) show that in the weakly nonlinear limit $(H-1 \ll 1) \Gamma_{K}$ is related to the speed along the wall of a trailing geostrophic current, $U_{\text {nose }}$, by

$$
\Gamma_{K}=1+\frac{1}{2} U_{\text {nose }} .
$$

For $U_{\text {nose }}>2$, the requirement (13) will be violated; however in this extreme case the weakly nonlinear conditions will probably be violated and (14) cannot be expected to hold. For example, examination of figure 15 in Helfrich et al. (1999) shows that (14) 
does not hold, yet the nose of the potential vorticity front trails the leading Kelvin shock. The assumption (13) will be checked in the numerical solutions ( $(3)$.

The semi-geostrophic relations (7) and (8), with $L_{0}$ replaced by $L$, also hold at all $x<x_{\text {nose }}(t)$ in the slowly varying downstream region. For $y \geqslant L, u=U(L(x, t)) \mathrm{e}^{-(y-L)}$, and therefore $h=1+U(L(x, t)) \mathrm{e}^{-(y-L)}$; thus

$$
U(L(x, t), t)=h(L(x, t), t)-1 \quad \text { at } \quad y=L(x, t)
$$

provides a boundary condition for the low-potential-vorticity $(y<L)$ fluid.

$$
\text { 2.2. Equations for } 0<y<L(x, t)
$$

To obtain equations for $U$ and $L$ the $y$-dependence of $u, h$ will be obtained from (7) and (8), and then substituted in the following relations. First we recall (Stern et al. 1982) that the conditions of piecewise-uniform potential vorticity and (3) imply that if the long-wave momentum equation (2) is satisfied at any one $y$ it is satisfied at all $y$. Therefore one equation which $U$ and $L$ must satisfy is obtained by setting $y=0$ and $v=0$ in (2):

$$
\frac{\partial u(x, 0, t)}{\partial t}+u(x, 0, t) \frac{\partial u(x, 0, t)}{\partial x}+\frac{\partial h(x, 0, t)}{\partial x}=0 .
$$

A second condition, obtained by integrating the continuity equation (4) from $y=0$ to $y=\infty$ and using $h=U \mathrm{e}^{-(y-L)}$ for $y>L$, and $v=0$ for $y=\infty$ is

$$
\int_{0}^{L} \frac{\partial h}{\partial t} \mathrm{~d} y+\int_{L}^{\infty} \frac{\partial}{\partial t}\left(U \mathrm{e}^{-(y-L)}\right) \mathrm{d} y+\frac{1}{2} \frac{\partial}{\partial x} h^{2}(x, 0, t)=0 .
$$

After using (15) this becomes

$$
\frac{\partial}{\partial t} \int_{0}^{L} h \mathrm{~d} y-\frac{\partial L}{\partial t}+\frac{\partial U}{\partial t}+\frac{1}{2} \frac{\partial}{\partial x} h^{2}(x, 0, t)=0 .
$$

\subsection{Zero potential vorticity}

The elimination of $(h, u)$ in (16) and (17) will be done first for the mathematically simplest case in which $q=H^{-1}=0$ in $y<L$, so that (8) gives $h$ as a parabolic function of $y$, and $u$ is linear, i.e.

$$
u(x, y, t)=U-(L-y), \quad h(x, y, t)=-U y-\frac{1}{2}(L-y)^{2}+\text { constant } .
$$

The latter constant, according to (15), is $1+U+L U$, and therefore

$$
\begin{gathered}
u(x, 0, t)=U-L, \quad h(x, 0, t)=1+U+L U-\frac{1}{2} L^{2}, \\
\int_{0}^{L} h \mathrm{~d} y=-\frac{L^{3}}{6}+\frac{U L^{2}}{2}+(1+U) L .
\end{gathered}
$$

Consequently (16) and (17) respectively become

$$
\begin{gathered}
\frac{\partial U}{\partial t}-\frac{\partial L}{\partial t}+\frac{\partial}{\partial x}\left(\frac{U^{2}}{2}+U\right)=0 \\
\frac{\partial}{\partial t}\left(U(1+L)+\frac{U L^{2}}{2}-\frac{L^{3}}{6}\right)+\frac{1}{2} \frac{\partial}{\partial x}\left(L U-\frac{L^{2}}{2}+1+U\right)^{2}=0 .
\end{gathered}
$$

We now seek similarity solutions of the form $L=L(U(x, t))$ which satisfy (10)-(13). 
(We note in passing that the quasi-linear hyperbolic equations (18) and (19) have an initial-value solution with spatially uniform $(L, U)$ at $x=-\infty$ such that the downstream state tends to evolve towards a Riemann invariant which is also of the form $L=L(U(x, t))$. This is one of the two aforementioned 'simple wave' solutions, and we shall try to satisfy the nose boundary condition with one of these.)

To obtain the simple wave we substitute $L=L(U)$ in (18) and (19) and denote $\mathrm{d} L / \mathrm{d} U$ by $L^{\prime}$ to obtain

$$
\left(1-L^{\prime}\right) \frac{\partial U}{\partial t}+(1+U) \frac{\partial U}{\partial x}=0
$$

and

$$
\begin{aligned}
\left(1+L+\frac{1}{2} L^{2}+U L^{\prime}\right. & \left.+U L L^{\prime}-\frac{1}{2} L^{2} L^{\prime}\right) \frac{\partial U}{\partial t} \\
& +\left(L U-\frac{1}{2} L^{2}+1+U\right)\left(L+1+U L^{\prime}-L L^{\prime}\right) \frac{\partial U}{\partial x}=0 .
\end{aligned}
$$

The solvability condition for $(\partial U / \partial t, \partial U / \partial x)$ in these quasi-linear relations then gives a quadratic equation for $L^{\prime}$ whose solutions are

$$
L^{\prime}(U)=\frac{-\beta \pm\left(\beta^{2}-4 \alpha c\right)^{1 / 2}}{2 \alpha},
$$

where

$$
\begin{aligned}
& \alpha=(U-L)\left(L U-\frac{1}{2} L^{2}+1+U\right), \\
& \beta=(2 L-U+1)\left(L U-\frac{1}{2} L^{2}+1+U\right)+(1+U)\left(U+U L-\frac{1}{2} L^{2}\right), \\
& c=-(L+1)\left(L U-\frac{1}{2} L^{2}+1+U\right)+(1+U)\left(1+L+\frac{1}{2} L^{2}\right) .
\end{aligned}
$$

The local propagation (characteristic) speed obtained from (20) is

$$
\Gamma=\frac{1+U}{1-L^{\prime}} .
$$

In order to determine the appropriate root of (22) for figure 1 we note that when $L \rightarrow 0, \alpha \rightarrow U(1+U), \beta \rightarrow 1+U$, and $c \rightarrow-L U \rightarrow 0^{-}$; thus the negative root

$$
L^{\prime}=\frac{-\beta-\left(\beta^{2}-4 \alpha c\right)^{1 / 2}}{2 \alpha},
$$

gives $L^{\prime} \rightarrow-\beta / \alpha=-U^{-1}$ as $L \rightarrow 0$, or

$$
\frac{\mathrm{d} L\left(U_{\text {nose }}\right)}{\mathrm{d} U}=-\frac{1}{U_{\text {nose }}},
$$

where $U_{\text {nose }}=U\left(x_{\text {nose }}, t\right)$. Since $U(L)$ decreases as $L$ increases from zero, this solution (27) is an 'expansion' wave, or 'wedge' intrusion. Furthermore (26) reduces to

$$
\Gamma_{\text {nose }}=U_{\text {nose }},
$$

which satisfies the important consistency requirement that the Lagrangian speed of the nose point equals the propagation speed of the point with constant $L=0$. On the other hand we may ignore the positive root of (22), which gives $\mathrm{d} L / \mathrm{d} U \rightarrow 0^{+}$ as $L \rightarrow 0$ (a 'bore' solution) because $\Gamma_{\text {nose }}=1+U_{\text {nose }}$ does not satisfy the above Lagrangian requirement at the nose.

When $H=\infty$, the upstream boundary condition (10) reduces to

$$
U\left(L_{0}\right)=L_{0},
$$




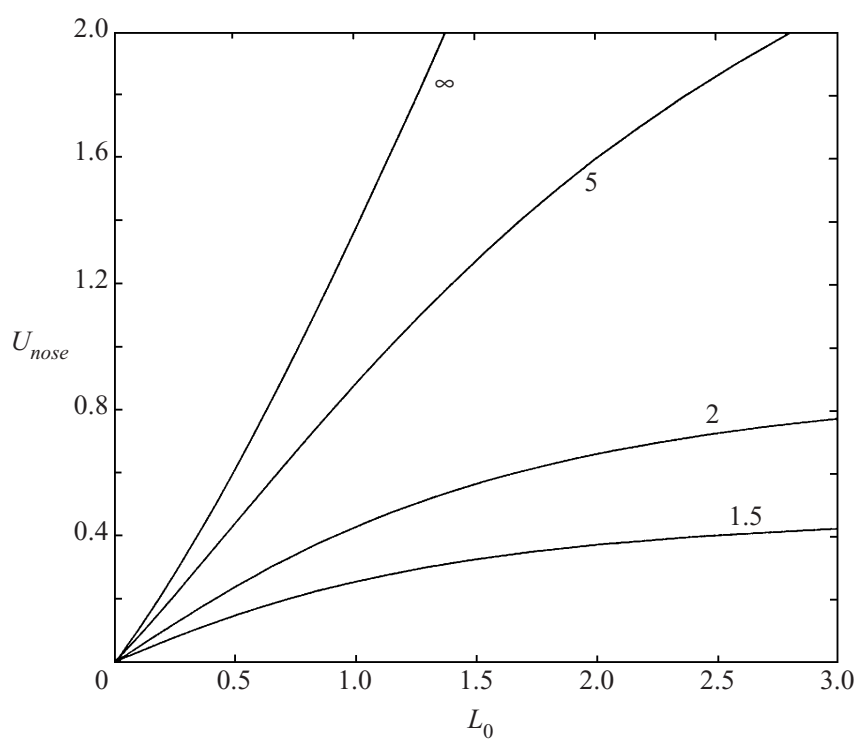

FIGURE 2. $U_{\text {nose }}$ versus $L_{0}$ for $H=1.5,2,5$ and $\infty$.

and the ordinary differential equation (27) will be solved with this boundary condition. We may integrate (27), starting from $L=0$ with any $U=U_{\text {nose }}$, and stopping when (30), or $U=L$, is satisfied; this gives $L_{0}=L, U_{0}=U$. Figure 2 shows the result of calculations of $U_{\text {nose }}$ as a function of $L_{0}$ for $H=\infty$, and in this limit $L_{*} / L_{0} \rightarrow 0$.

\subsection{Finite potential vorticity}

Now we turn to the more realistic case of finite $H$.

For any $L$ in equations (7) and (8) the solutions which satisfy the boundary conditions $u(x, L, t)=U, h(x, L, t)=1+U$ are

$$
\begin{gathered}
h=A \cosh \left(y / H^{1 / 2}\right)+B \sinh \left(y / H^{1 / 2}\right)+H, \\
u=-A H^{-1 / 2} \sinh \left(y / H^{1 / 2}\right)-B H^{-1 / 2} \cosh \left(y / H^{1 / 2}\right),
\end{gathered}
$$

where

$$
\begin{aligned}
& A=H^{1 / 2} U \sinh \left(L / H^{1 / 2}\right)+(1+U-H) \cosh \left(L / H^{1 / 2}\right), \\
& B=-H^{1 / 2} U \cosh \left(L / H^{1 / 2}\right)-(1+U-H) \sinh \left(L / H^{1 / 2}\right) .
\end{aligned}
$$

In (16) and (17) we now substitute $h(x, 0, t)=H+A, u(x, 0, t)=-B H^{-1 / 2}$, and

$$
\int_{0}^{L} h \mathrm{~d} y=H L+A H^{1 / 2} \sinh \left(L / H^{1 / 2}\right)+B H^{1 / 2}\left(\cosh \left(L / H^{1 / 2}\right)-1\right)
$$

to obtain

$$
\begin{gathered}
-H^{-1 / 2} \frac{\partial B}{\partial t}+B H^{-1} \frac{\partial B}{\partial x}+\frac{\partial A}{\partial x}=0 \\
\frac{\partial}{\partial t}\left[U-L+H L+A H^{1 / 2} \sinh \left(L / H^{1 / 2}\right)+B H^{1 / 2}\left(\cosh \left(L / H^{1 / 2}\right)-1\right)\right] \\
+(H+A) \frac{\partial A}{\partial x}=0 .
\end{gathered}
$$


Equations (33)-(36) determine the evolution of $L(x, t), U(x, t)$. We again seek a similarity solution of the form $L=L[U(x, t)]$, for which the following relations are satisfied:

$$
\begin{gathered}
\frac{\partial(\cdot)}{\partial t}=\frac{\partial U}{\partial t} \frac{\partial(\cdot)}{\partial U}+\frac{\partial L}{\partial t} \frac{\partial(\cdot)}{\partial L}=\frac{\partial U}{\partial t}\left(\frac{\partial(\cdot)}{\partial U}+L^{\prime}(U) \frac{\partial(\cdot)}{\partial L}\right), \\
\frac{\partial(\cdot)}{\partial x}=\frac{\partial U}{\partial x}\left(\frac{\partial(\cdot)}{\partial U}+L^{\prime}(U) \frac{\partial(\cdot)}{\partial L}\right) .
\end{gathered}
$$

Equations (35) and (36) then give two quasi-linear equations,

$$
\begin{aligned}
& \left(a_{11}+b_{11} L^{\prime}\right) \frac{\partial U}{\partial t}+\left(a_{12}+b_{12} L^{\prime}\right) \frac{\partial U}{\partial x}=0, \\
& \left(a_{21}+b_{21} L^{\prime}\right) \frac{\partial U}{\partial t}+\left(a_{22}+b_{22} L^{\prime}\right) \frac{\partial U}{\partial x}=0,
\end{aligned}
$$

where the coefficients $a_{11} \ldots b_{22}$ are functions of $(U, L)$ as given in the Appendix. The determinant of the above system yields a quadratic equation for $L^{\prime}$,

$$
\alpha\left(L^{\prime}\right)^{2}+\beta L^{\prime}+c=0,
$$

one of whose roots is

$$
\begin{gathered}
L^{\prime}(U)=\frac{-\beta-\left(\beta^{2}-4 \alpha c\right)^{1 / 2}}{2 \alpha} \equiv \frac{1}{F}, \\
\frac{\mathrm{d} U}{\mathrm{~d} L}=F(U, L),
\end{gathered}
$$

where the new $\alpha, \beta$ and $c$ are also given in the Appendix.

The negative root (40) of the quadratic was again chosen, for the following reason. When $L \rightarrow 0$ it is easily shown that $a_{11} \rightarrow 1, b_{11} \rightarrow U, a_{12} \rightarrow 1+U, b_{12} \rightarrow$ $U(1+U), a_{21} \rightarrow 1, b_{21} \rightarrow(1+U-H) / H, a_{22} \rightarrow 1+U$, and $b_{22} \rightarrow U(1+U) / H$. Thus $\left(a_{11} a_{22}-a_{12} a_{21}\right) \rightarrow 0$ and the non-vanishing root of the quadratic is $L^{\prime}(U) \rightarrow-U^{-1}$, so that the propagation speed

$$
\Gamma=\frac{a_{22}+b_{22} L^{\prime}}{a_{21}+b_{21} L^{\prime}} \rightarrow U .
$$

This property of the hyperbolic equations (37) and (38) therefore agrees with the boundary condition $\mathrm{d} x_{\text {nose }} / \mathrm{d} t=U(0)=U_{\text {nose }}$. The positive root of the quadratic, on the other hand, gives $L^{\prime} \rightarrow 0$ and $\Gamma \rightarrow 1+U$, which violates the aforementioned boundary condition. If $L$ increases as $x-x_{\text {nose }}$ decreases, then (40) implies that $U$ decreases, and an 'expansion' wave is again obtained.

Curves of $U_{\text {nose }}$ as functions of $L_{0}$ are obtained by numerically integrating (41) starting with any $U=U_{\text {nose }}$ at $L=0$ and stopping the integration when the upstream boundary condition (10) is satisfied, at which point $(L, U)$ we set $L_{0}=L$ and $U_{0}=U$; $L_{*}$ is then computed from (11). For several values of $H$ the functional relationship between $L_{0}$ and $U_{\text {nose }}$ is plotted in figure 2, and between $L_{0}$ and $L_{*}$ in figure 3. In figure 2 it appears that for $H=1.5$ and $H=2$ the value of $U_{\text {nose }}$ asymptotes to a constant as $L_{0} \rightarrow \infty$, as was verified by numerical integration of (41). The asymptotic value $U_{\text {nose }}^{\infty}=U_{\text {nose }}\left(L_{0} \rightarrow \infty\right)$ is shown in figure 4 . 


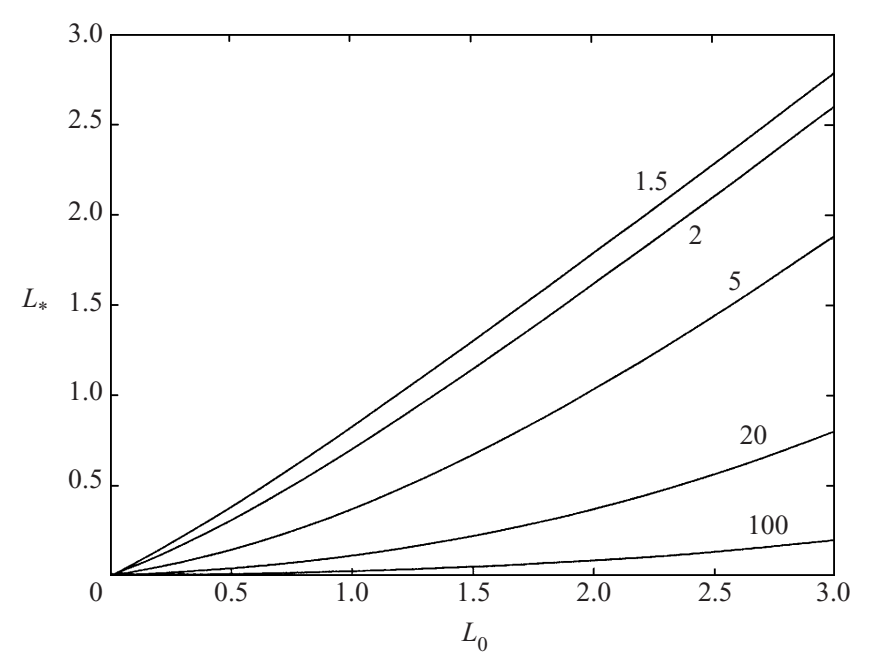

FiguRe 3. $L_{*}$ versus $L_{0}$ for $H=1.5,2,5,20$ and 100 .

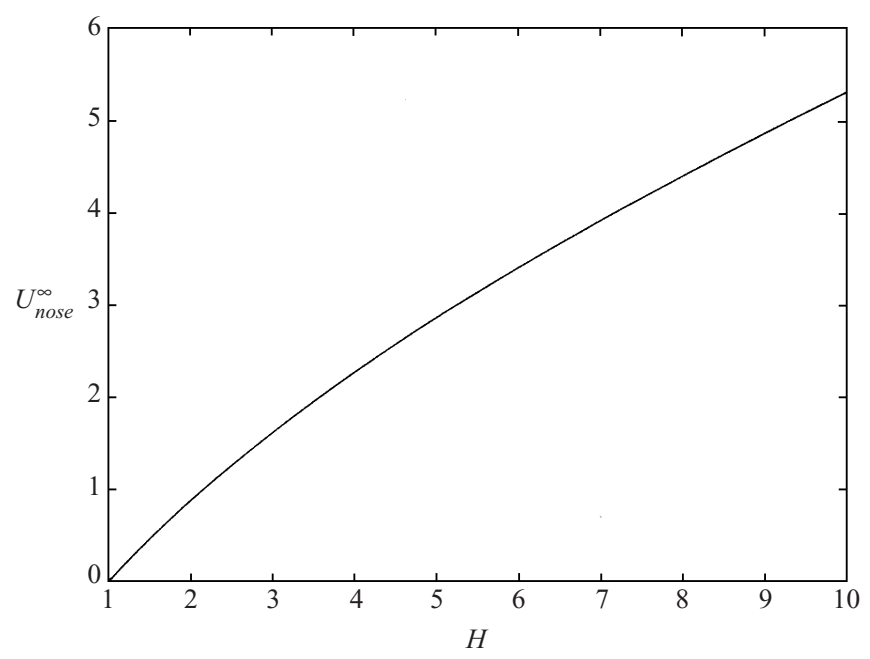

FIGURE 4. The asymptotic nose speed $U_{\text {nose }}^{\infty}$ (for $L_{0} \rightarrow \infty$ ) as a function of $H$.

\section{Numerical study}

Our long-wave theory for the intruding potential vorticity front relies on several questionable assumptions. Obviously the restriction to long spatial scales will not be valid immediately after the removal of the dam depicted in figure 1, and the shortwave disturbances may affect the long time evolution. The theory also singles out the expansion wave solution based on an assumption that the leading Kelvin shock speed $\Gamma_{K}>U_{\text {nose }}$. This is a reasonable assumption based on the numerical results of Herman et al. (1989) and Helfrich et al. (1999), and the weakly nonlinear analysis of Fedorov \& Melville (1996), but should be tested in this context. The theory also assumes that the leading Kelvin wave does not modify the potential vorticity of the ambient fluid which lies between the intrusion nose and the Kelvin wave. However, in the initial-value problem posed in figure 1 the leading Kelvin wave immediately steepens into a shock (Fedorov \& Melville 1996; Helfrich et al. 1999) across which 
potential vorticity is not necessarily conserved (Pratt 1983; Helfrich et al. 1999). This alteration may affect the intrusion speed and structure. To examine these issues we have conducted a numerical study of the dam-break problem using a shock-capturing shallow-water equation model.

\subsection{Numerical models}

The main numerical results are based on a solution of the reduced-gravity ( $1 \frac{1}{2}$-layer) non-dimensional shallow-water equations in conservation form,

$$
\begin{gathered}
\frac{\partial(u h)}{\partial t}+\frac{\partial}{\partial x}\left(u^{2} h+\frac{1}{2} h^{2}\right)+\frac{\partial}{\partial y}(u v h)-v h=v \nabla \cdot(h \nabla u), \\
\frac{\partial(v h)}{\partial t}+\frac{\partial}{\partial x}(u v h)+\frac{\partial}{\partial y}\left(v^{2} h+\frac{1}{2} h^{2}\right)+u h=v \nabla \cdot(h \nabla v), \\
\frac{\partial h}{\partial t}+\frac{\partial(u h)}{\partial x}+\frac{\partial(v h)}{\partial y}=0 .
\end{gathered}
$$

Here $u$ and $v$ are the horizontal velocities in the $x$ - and $y$-directions, respectively, and $v$ is a constant eddy viscosity. As in $\S 2$, the velocities $u$ and $v$ are scaled by $\sqrt{g^{\prime} H_{0}}$, the layer depth $h$ with $H_{0}$, horizontal dimensions $(x, y)$ with the deformation radius $L_{D}=\sqrt{g^{\prime} H_{0}} / f$, and time $t$ by $f^{-1}$. These equations are solved in conservation form to ensure discrete mass and momentum conservation across any Kelvin shocks. The numerical model is the one used in Helfrich et al. (1999), where details of the numerical scheme and model testing are discussed. In summary, the model solves (43), (44) and (45) using a cell-centred, slope-limited scheme for the advective fluxes (LeVeque 1997). The Coriolis, pressure and dissipation terms are handled with centred differences and Strang splitting in time. The model is second-order in space and time, except near shocks where the spatial order may be reduced due to the slope limiting of the advective fluxes.

The initial conditions,

$$
\begin{gathered}
h= \begin{cases}H, & x<0 \text { and } 0 \leqslant y<L_{*} \\
1, & \text { otherwise }\end{cases} \\
u=v=0 \text { everywhere, }
\end{gathered}
$$

are illustrated in figure 1. The extent of model domain is $X_{\min } \leqslant x \leqslant X_{\max }$ and $0 \leqslant$ $y \leqslant Y_{\max }$. At $x=X_{\min }$ the boundary condition is $\partial / \partial x=0$. Provided $\left|X_{\min }\right| / L_{*} \gg 1$ this is a reasonable approximation since it will take a finite time for any disturbances from the dam at $x=0$ to propagate upstream. (In the long-wave limit all disturbances have positive phase speeds so only short-wave disturbances are expected to propagate upstream.) At the downstream boundary $x=X_{\max }$ and at $y=Y_{\max }$ open radiation conditions are employed (Orlanski 1969). In the calculations presented here the model is run with no explicit viscosity, $v=0$. Thus the $y=0$ boundary condition is just $v=0$. The model is solved on a rectangular grid with cell spacing $\Delta x=\Delta y=0.1$ or 0.05 . The time step $\Delta t=0.02$ and 0.01 , respectively. In most calculations $X_{\min }=-35$, $X_{\max }=35$, and $Y_{\max }=8$.

In the inviscid limit the potential vorticity $q$ is conserved following fluid parcels. However, $q$ is not conserved across a Kelvin shock (Pratt 1983; Helfrich et al. 1999) and this alteration may affect the intrusion of the trailing potential vorticity front. Also, the non-conservation of potential vorticity implies that $q$ may not differentiate between fluid initially behind the dam $\left(q=H^{-1}\right)$ and the ambient fluid $(q=1)$. Thus 
to distinguish these fluid parcels we solve an equation for a conservative tracer with concentration $c$,

$$
\frac{\partial(c h)}{\partial t}+\frac{\partial(u c h)}{\partial x}+\frac{\partial(v c h)}{\partial y}=0,
$$

with initial condition $c=1$ for fluid behind the dam and $c=0$ elsewhere. The $c=0.5$ contour is treated as the material surface between these regions and compared to both the calculated $q$ field and the long-wave theory, $L(x, t)$, for the potential vorticity front.

Numerical model estimates of the potential vorticity intrusion nose speed $U_{\text {nose }}$ are obtained by first interpolating in $x$ for the position of the $c=0.5$ contour in the first two cells adjacent to the $y=0$ wall (centred at $y=\frac{1}{2} \Delta y$ and $\frac{3}{2} \Delta y$ ). These values are then used to linearly extrapolate the $x$ location of the $c=0.5$ contour to $y=0$, which is identified as the numerical nose position $x_{\text {nose }}(t)$. $U_{\text {nose }}$ is obtained by fitting a line to the discrete $x_{\text {nose }}(t)$ data. Differencing the discrete $x_{\text {nose }}(t)$ data gives nearly identical mean propagation speeds.

For comparison with the laboratory experiments we also made runs using a reservoir with finite length in $x$ and periodic boundary conditions in $x$. Additionally, some calculations have been made with a two-layer free-surface version of the shallowwater numerical model. The numerical methods are the same as described above. Unless specifically noted all results are from the single-layer (i.e. reduced-gravity) model.

\subsection{Numerical results}

The evolution of the flow for a case with $H=2$ and $L_{0}=1.881\left(L_{*}=1.5\right)$ is given in figure 5 . The Kelvin shock quickly outruns the potential vorticity intrusion, which advances as an expansion as predicted. The dam removal also generates a short-wave disturbance which propagates back upstream without affecting the location of the potential vorticity front. At $t=25$ the average front position far upstream of the dam $(x<-10)$ is $L=1.881$, in excellent agreement with the theory $L_{0}=1.881$. The amplitude of the Kelvin shock along the right-hand wall is 0.6 giving a total fluid depth of 1.6. Details of the dynamics of Kelvin shocks are discussed in Fedorov \& Melville (1996) and Helfrich et al. (1999). The potential vorticity $q$ along the wall $y=0$ at $t=25$ (figure $5 d$ ) between the intrusion nose $x_{\text {nose }}=15.0$ and the Kelvin bore $x=33.5$ has been altered from the initial upstream value. The alteration is confined to a narrow strip near the boundary and the structure of the change is in agreement with theoretical expectations of rapid decay of the alteration with jump height and the sign of the potential vorticity modification (see Pratt 1983). The nose position $x_{\text {nose }}$ for this run is shown in figure $6(a)$. The nose quickly accelerates to reach a nearly uniform speed of $U_{\text {nose }}=0.63$ for $t>7$. The slight tendency for $U_{\text {nose }}$ to decrease for $t>22$ (figure $6 b$ ) is not due to the downstream radiation boundary. It may be a slight effect of the alteration of the upstream potential vorticity field.

A comparison of the similarity solution $L(x, t)$ and the numerical solution for the potential vorticity front (from the $c=0.5$ contour) is made in figure 7 for the run in figure 5. Rather than initialize the theory with the right-angle dam shape, the numerical solution at $t=5$ and $x>-2.5$ is smoothly matched to the theoretical upstream solution $\left(L_{0}=1.881\right)$ and used as the initial condition. This is a fairer test of the theory since it is formally valid at times after the dam removal when the far-field geostrophic flows have been established. With this initial condition the agreement between the numerical model and the theory is very good. In fact, if the 

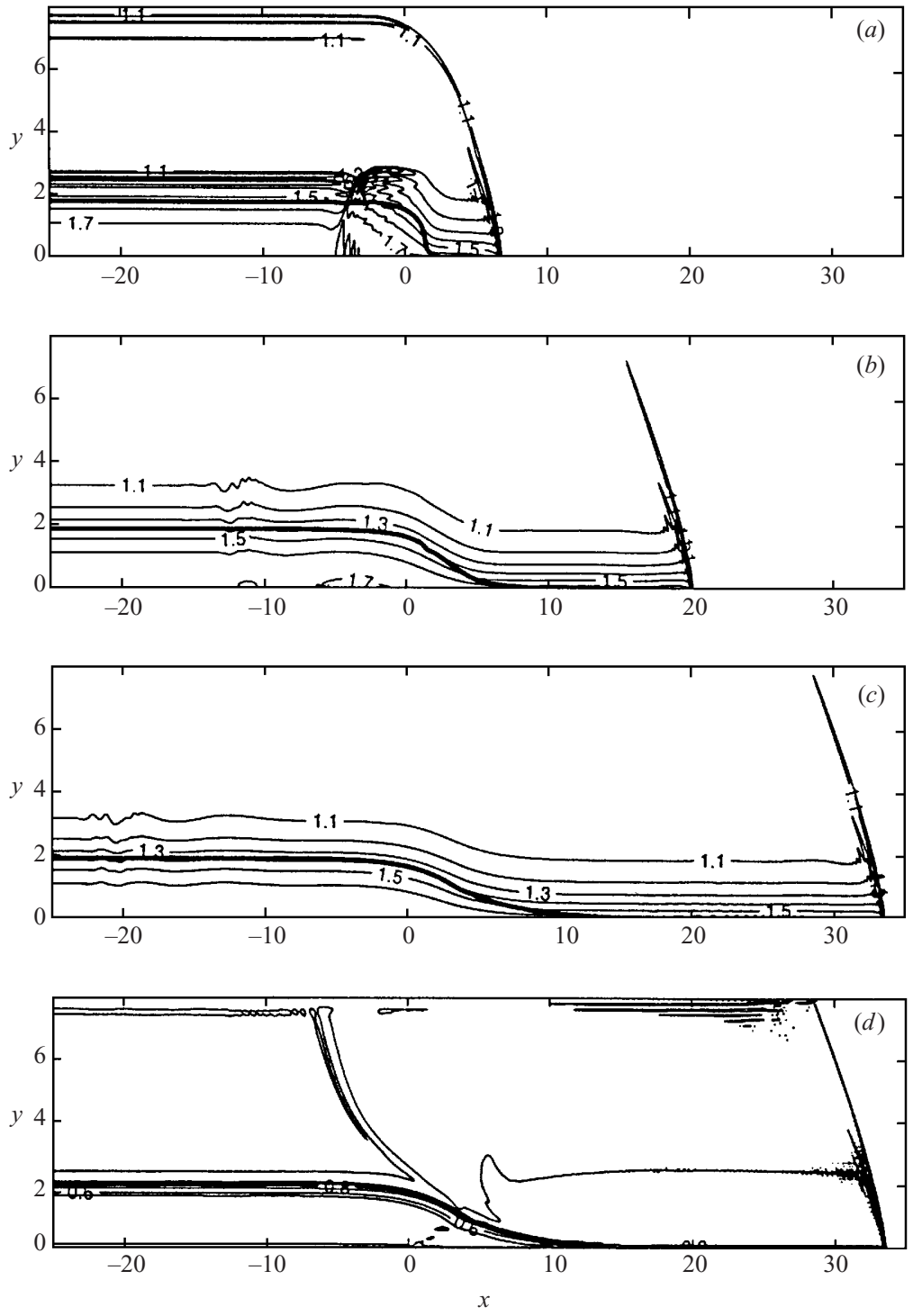

Figure 5. Numerical solution for $H=2$ and $L_{*}=1.5\left(L_{0}=1.88\right)$. Contours of the layer depth $h$ (contour interval of 0.1 ) at $(a) t=5,(b) t=15$ and $(c) t=25$. The heavy solid line is the $c=0.5$ contour which defines the intrusion boundary. Contours of potential vorticity $q$ at $t=25$ are plotted in $(d)$. In this run $\Delta x=\Delta y=0.05$. Note that the left-hand boundary $X_{\min }=-35$ is not shown.

dam shape is naively used as the initial condition for the similarity theory the front shape near the nose at $t=25$ is still in good agreement with the numerical model as indicated in figure 7.

The numerically computed $U_{\text {nose }}$ is plotted along with the theoretical results for $H=1.5,2$ and 5 in figure 8 . The agreement is very good for $H=1.5$ and 2 and independent of the two model resolutions tested. For $H=5$ the numerical speeds are $5-15 \%$ below the theory. This disagreement may be a consequence of the upstream potential vorticity alteration by the Kelvin bore. For $H=5$ the bore amplitudes at the wall are very large $(\approx 2.5)$, while the transverse decay scale remains proportional 

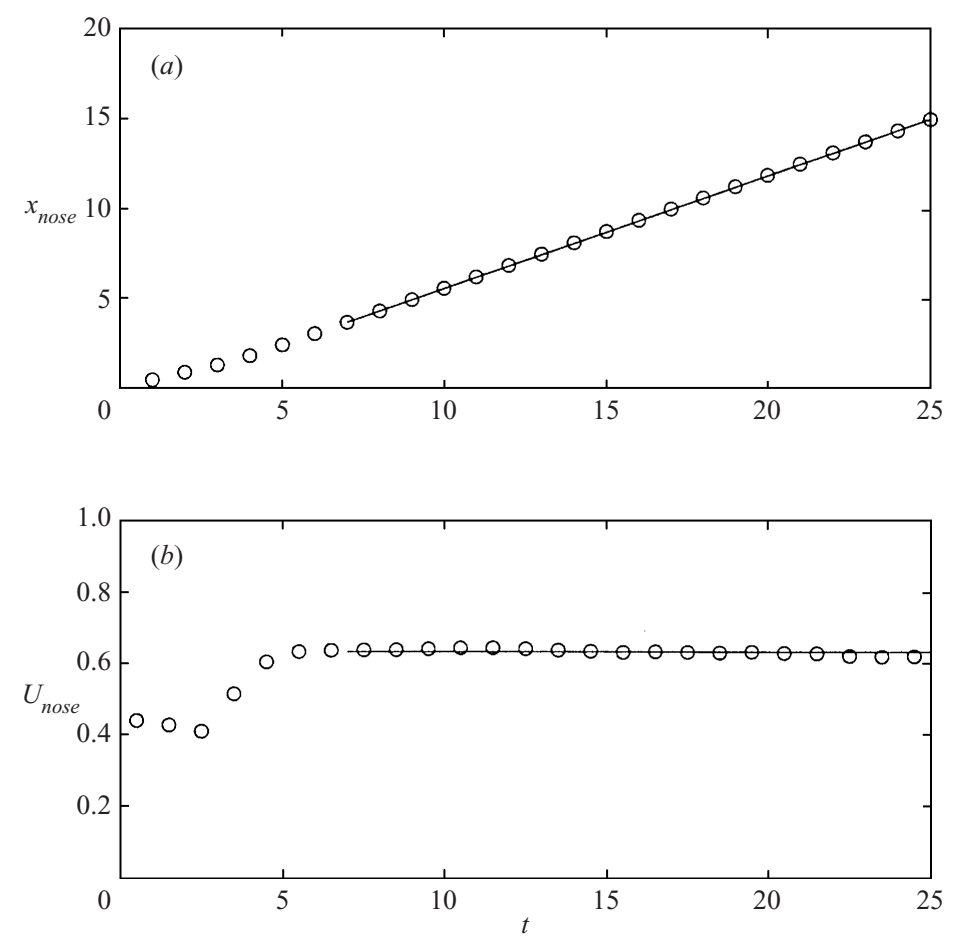

FiguRE 6. (a) $x_{\text {nose }}$ versus $t$ for the numerical run in figure 5 . The solid line is the linear fit to the data for $t \geqslant 7$ and gives $U_{\text {nose }}=0.633$. (b) $U_{\text {nose }}$ versus $t$ with $U_{\text {nose }}$ determined from differencing $x_{\text {nose }}(t)$. The line is the mean speed $U_{\text {nose }}=0.632$ for $t>7$.

to the upstream $q$ value. As a result the potential vorticity change near the wall, which is proportional to the transverse gradient of the cube of the jump amplitude, increases with $H$ (see Helfrich et al. 1999 for examples). Despite the departure in the numerical nose speeds for $H=5$, the expansion structure of the front remains.

\section{Experimental study}

As a test of the theory and numerical results some simple laboratory experiments were conducted. The goal was to examine the structure and speed of the potential vorticity intrusion. To make the experiments tractable they are carried out in a two-layered configuration with the lower layer much deeper than the upper 'active' layer in the reduced-gravity approximation. However, the presence of this lower layer introduces the possibility of non-hydrostatic interfacial instabilities resulting in vertical mixing and baroclinic instabilities which may affect the evolution.

\subsection{Experimental methods}

The experiments were carried out in a rotating $2.1 \mathrm{~m}$ diameter cylindrical tank in the Geophysical Fluid Dynamics Laboratory at the Woods Hole Oceanographic Institution (figure 9). The tank was first filled to a depth $H_{0} \mathrm{~cm}$ with salt water of density $\rho_{1}$ and brought into counterclockwise solid body rotation at rate $\Omega=f / 2$. Saltwater of density $\rho_{2}\left(>\rho_{1}\right)$ was then slowly added over a period of several hours through a diffuser on the tank bottom until a depth of slightly less than the target total depth $H_{T}$ was achieved. The densities were measure with an Anton Paar model 


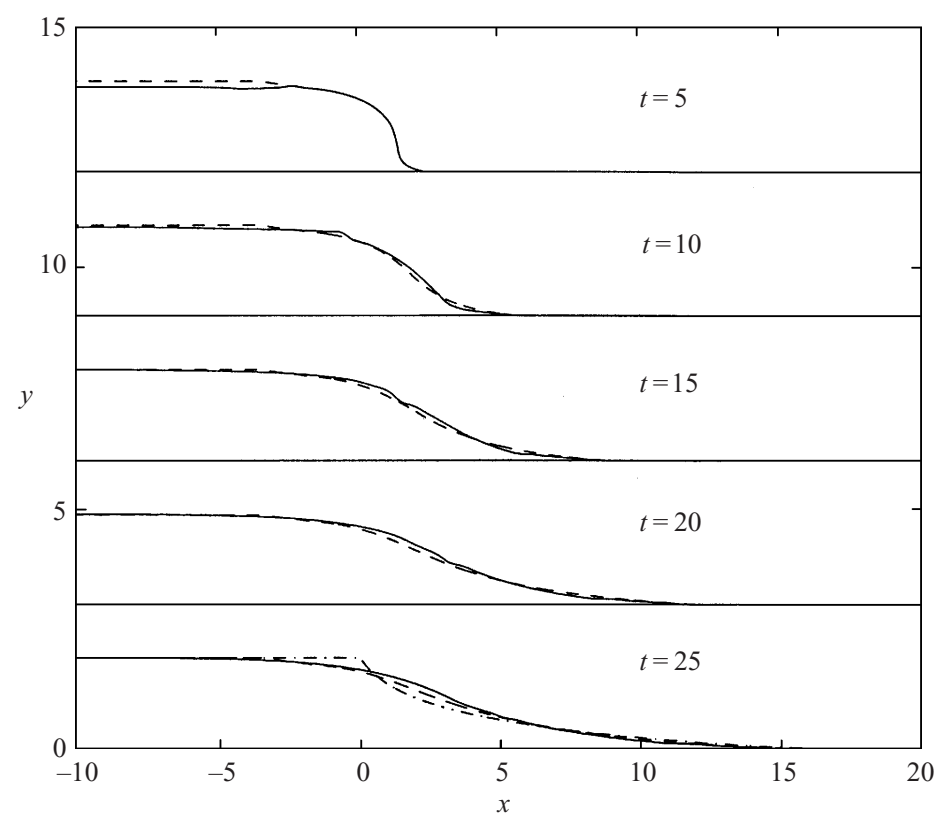

FIGURE 7. Comparison of the theoretical solution (dashed line) with the numerically calculated intrusion boundary ( $c=0.5$ contour-solid line) for the run in figure 5 . The leading region of the numerical solution $(x>-2.5)$ at $t=5$ is matched to the analytical upstream state $\left(L_{0}=1.881\right)$ and used as the initial condition for the theoretical solution for $t>5$. The dash-dot line at $t=25$ is the similarity solution using the dam shape at $t=0$ as the initial condition. The times are indicated and the curves are offset in $y$ by three units.

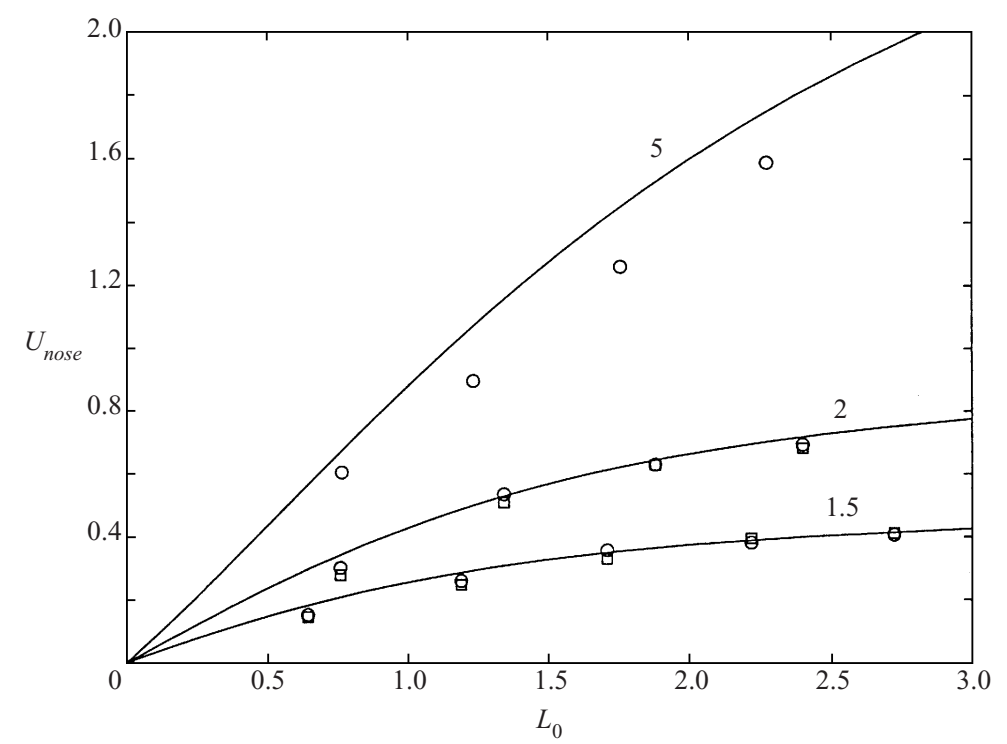

FIgURE 8. Numerical $U_{\text {nose }}$ versus $L_{0}$. The symbols are from the numerical model with resolution $\Delta x=0.05$ (circles) and $\Delta x=0.1$ (squares). The lines are the theory for $H=1.5,2$ and 5 . 

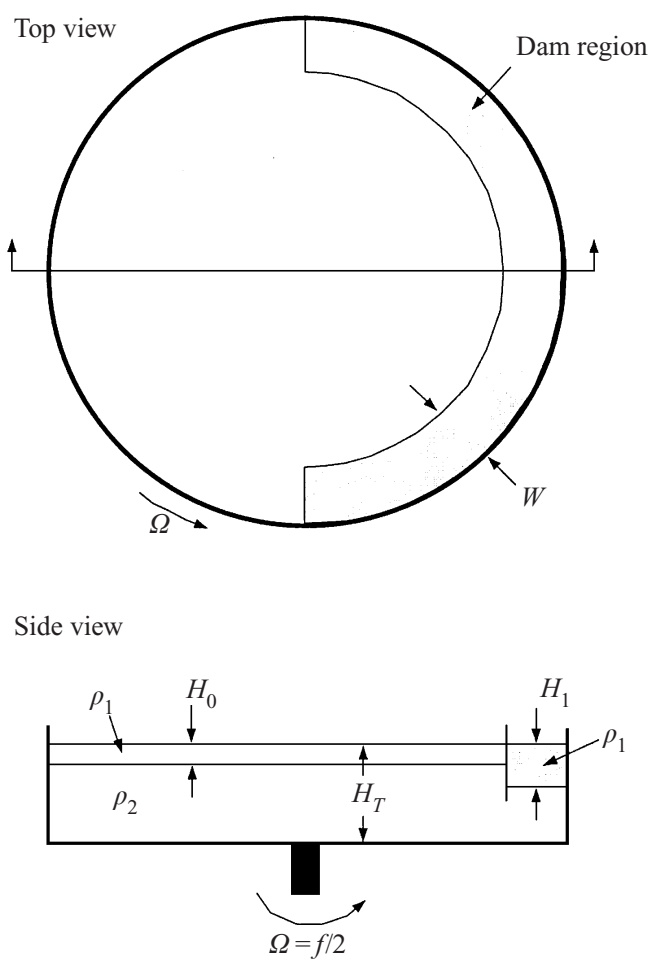

FIGURE 9. Experimental setup.

DMA58 densitometer with accuracy of $10^{-5} \mathrm{~g} \mathrm{~cm}^{-3}$. During the filling procedure the water rose up into a semi-circular dam with uniform width $W$ situated against the tank wall. Additional upper-layer water $\left(\rho_{1}\right)$ was then added behind the dam to bring the dammed upper-layer depth to $H_{1}$ and the total depth to $H_{T}$. The added water was dyed dark blue for visualization and gently mixed with the pre-existing light water behind the dam. In the absence of interfacial mixing the dye is analogous to the tracer field used in the numerical model. During the filling stage the tank is covered to eliminate wind drag. The covered tank is then allowed to spin-up for two hours until near solid body rotation was achieved. Residual flows were $O\left(0.1 \mathrm{~cm} \mathrm{~s}^{-1}\right)$, well below the expected intrusion speed of $O\left(5 \mathrm{~cm} \mathrm{~s}^{-1}\right)$. The experiment was initiated by rapidly, and as carefully as possible, lifting the dam. The evolution of the dyed water was viewed from above with a co-rotating black and white CCD camera and the images were directly digitized and saved to to a computer at intervals of 1 or $2 \mathrm{~s}$. The camera signal was also recorded by a VCR.

There were seven experimental runs (table 1). The dam width was $W=18 \mathrm{~cm}$ for all runs and the Coriolis frequency was $f=0.35-1 \mathrm{~s}^{-1}$. The experiments were scaled using the linear two-layer Kelvin wave speed $c_{2}=\left(g^{\prime} H_{0}\left(H_{T}-H_{0}\right) / H_{T}\right)^{1 / 2}$ based on the ambient stratification and the corresponding two-layer deformation radius $L_{D 2}=c_{2} / f$; the reduced gravity $g^{\prime}=g\left(\rho_{2}-\rho_{1}\right) / \rho_{2}$, where $g$ is the acceleration due to gravity. These give scaled dam widths $L_{*}=W / L_{D 2}=0.5-2.5$, and scaled depth $H=H_{1} / H_{0}=1.5$ and 2 behind the dam. Quantitative observations were made of the intrusion nose speed $U_{\text {nose }}^{\text {obs }}$ and the offshore location of the upstream potential vorticity front $L_{0}^{o b s}$. The speed is obtained from time series of the observed nose position $x_{n o s e}^{o b s}$, defined as the circumferential distance of the farthest advance of the 


\begin{tabular}{cccccccccccc}
\hline Run & $H_{0}$ & $H_{1}$ & $H_{T}$ & \multicolumn{1}{c}{$g^{\prime}$} & \multicolumn{1}{c}{$f$} & $W$ & $c_{2}$ & $L_{D 2}$ & $U_{n o s e}^{o b s}$ & $L_{0}^{o b s}$ & $\Gamma_{K}^{o b s}$ \\
1 & 7 & 14 & 38.2 & 12.70 & 0.50 & 18 & 8.5 & 17.0 & 4.8 & 30.2 & 10.5 \\
2 & 7 & 14 & 38.0 & 10.96 & 1.00 & 18 & 7.9 & 7.9 & 3.5 & 23.4 & 10.6 \\
3 & 7 & 14 & 38.0 & 22.57 & 0.35 & 18 & 11.4 & 32.4 & 3.9 & 33.1 & 14.0 \\
4 & 7 & 10.5 & 38.2 & 12.05 & 0.50 & 18 & 8.3 & 16.6 & 2.0 & 21.7 & 9.9 \\
5 & 7 & 10.5 & 37.8 & 9.42 & 1.00 & 18 & 7.3 & 7.3 & 2.1 & 21.6 & 9.0 \\
6 & 7 & 10.5 & 38.0 & 22.71 & 0.35 & 18 & 11.4 & 32.5 & 4.0 & 27.3 & 9.3 \\
7 & 7 & 14 & 38.2 & 26.97 & 1.00 & 18 & 12.4 & 12.4 & 5.3 & 27.1 & 14.1 \\
\multicolumn{10}{c}{ TABLE 1. Parameters and results for laboratory runs. All data in c.g.s. units. } \\
\hline
\end{tabular}

dyed fluid along the wall from the position of the dam $(x=0) . U_{\text {nose }}^{o b s}$ is determined by fitting a line through the $x_{n o s e}^{o b s}(t)$ data (see figures 10 and 11). The width $L_{0}^{o b s}$ is obtained by contouring the greyscale images and averaging the radial position of the contour defining the dye boundary over the middle section of the front, away from the intrusion nose and the upstream end. A sequence of images in a run gives a time series of widths, which are averaged in time to produce a time- and space-averaged value. The temporal averaging is necessary to reduce the effects of inertial oscillations which are excited when the dam is removed, and the spatial averaging smooths wave-like disturbances on the front. The speed of the leading Kelvin shock $\Gamma_{K}^{o b s}$ (which is not directly visible in the images) is estimated from the time between the dam removal and the arrival of the disturbance at the upstream end of the low-potential-vorticity front (see $§ 4.2)$.

\subsection{Experimental results}

Images from run $7\left(H=2\right.$ and $\left.L_{*}=W / L_{D 2}=1.4\right)$ are shown in figure 10. Immediately after the dam is removed the dyed fluid slumps away from the tank wall and surges forward near the head of the dam (panel $a$ ). Shortly afterward the intrusion begins to propagate counterclockwise along the wall as an expansion (panels $b, c$ ). The outer edge of the intrusion and the upstream geostrophic current experience an instability (panels $c, d$ ) that at finite amplitude results in backward breaking waves (panels $e-h$ ). The leading Kelvin wave is not directly observable in these images. However, the arrival of the Kelvin wave at the trailing edge of the region of dyed (low-potential-vorticity) fluid rapidly forces this fluid away from the wall (panels $c, d$ ). This generates a vortex pair that moves into the tank interior (panels $e-h$ ). This process is discussed further in $\S 5$. The important point is that this occurs prior to the arrival of the nose of the intrusion (panel $h$ ) and is associated with the leading Kelvin bore.

The nose position in time is characterized by three stages (figure 11). Over the first $5-10 \mathrm{~s}$ the nose rapidly advances at a nearly constant speed. This is followed by a longer period of uniform, but slower, advance. In the third stage the nose may stagnate, then may accelerate again as seen clearly for runs 3 and 6 in figure 11 . The stagnation and reacceleration is due to the finite length of the dammed region and the azimuthally periodic structure of the experiment. This is discussed further in $\S 5$. The initial rapid advance is probably related to the initial gravity-dominated adjustment before rotational effects become significant. Thus, we use the speed determined in the second stage as the intrusion speed $U_{\text {nose }}^{\text {obs }}$ to compare with the theory. Interestingly, in the experiments the initial stage has a larger speed than the second stage, while in the numerical results the initial adjustment is characterized by speeds less than the final 


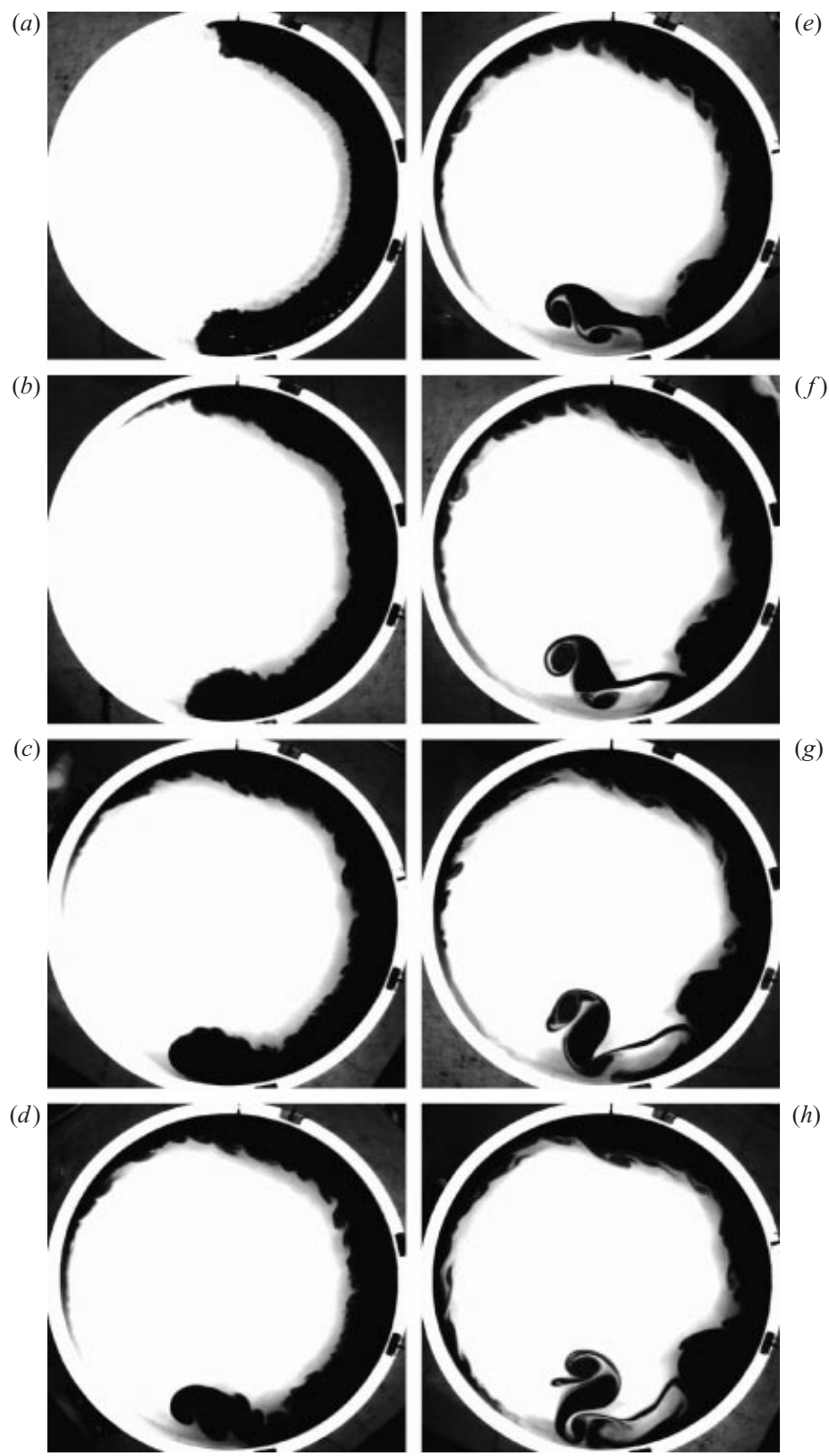

FIGURE 10. Images from run 7 with $H=2$ and $W / L_{D 2}=1.4$. (a) $t=3 \mathrm{~s},(b) 9 \mathrm{~s},(c) 23 \mathrm{~s}$, (d) $33 \mathrm{~s}$, (e) $43 \mathrm{~s},(f) 53 \mathrm{~s},(g) 63 \mathrm{~s},(h) 73 \mathrm{~s}$.

intrusion speed (see figure 6). This may be a consequence of non-hydrostatic effects and the finite time for the dam removal not accounted for in the numerical model.

The experimental nose speeds agree only qualitatively with the theory (figure 12). In the figure, $U_{\text {nose }}^{o b s}$ is scaled by the two-layer Kelvin wave speed $c_{2}$ and is plotted against the similarly scaled dam width $L_{*}=W / L_{D 2}$, rather than the final upstream geostrophic front width $L_{0}$, since $L_{*}$ is the independent experimental parameter. The speeds for $H=2$ are larger than for $H=1.5$ as predicted, but for both values of 

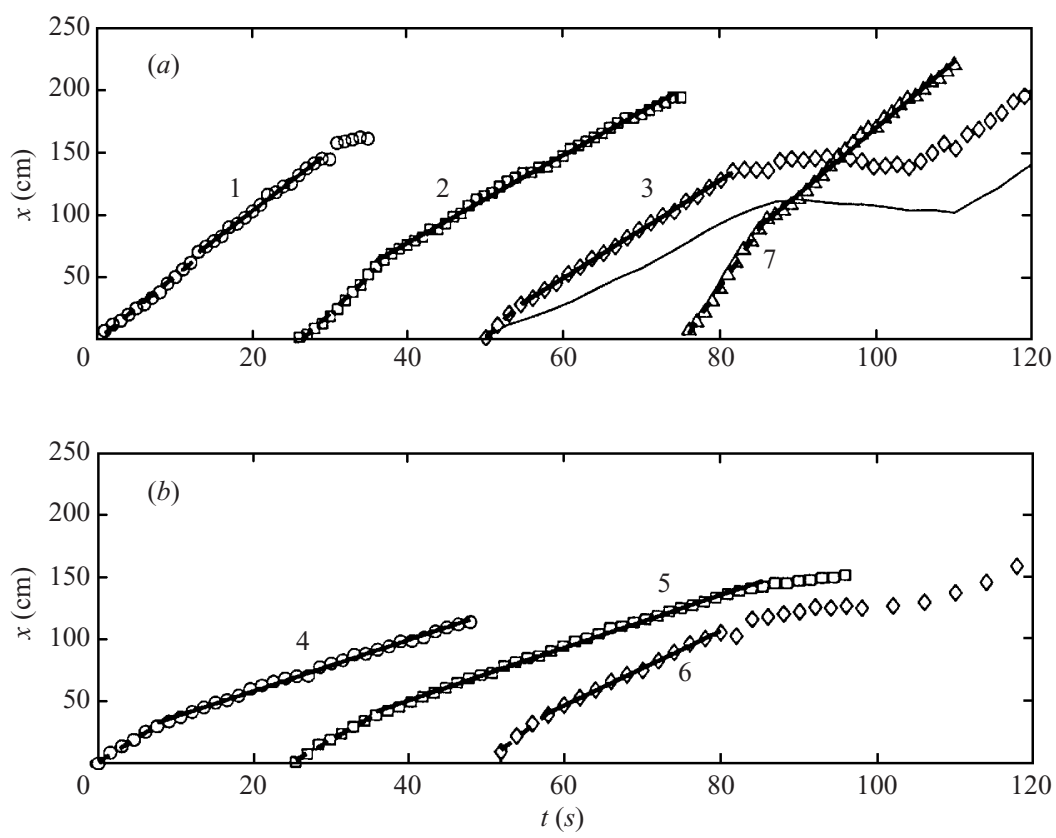

FiguRE 11. Experimental $x_{\text {nose }}$ versus $t$ for $H=2(a)$, and $H=1.5(b)$. The run number is indicated next to each set of data and each run is offset by $25 \mathrm{~s}$. The thick dashed line is the best linear fit to the first stage and the thick solid line is the best linear fit to the second stage. The length of each stage in a run is indicated by the endpoints of the lines. The slope of the thick solid line gives $U_{\text {nose }}^{o b s}$. The thin solid line adjacent to run 3 is the result from the two-layer numerical model (discussed in $\S 5)$.

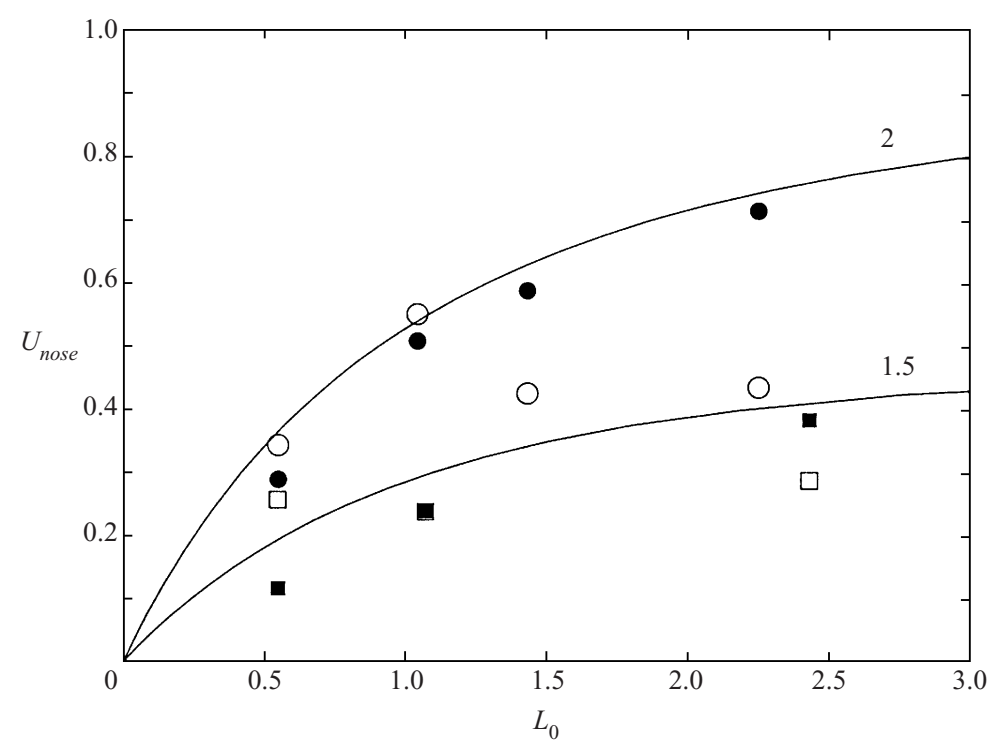

FIGURE 12. Experimental $U_{\text {nose }}$ versus $L_{*}$. The open symbols are for the experiments with $H=2$ (circles) and $H=1.5$ (squares). The solid symbols are from the two-layer numerical model run with experimental parameters. The experimental data and the two-layer model results are scaled by the two-layer linear phase speed $c_{2}=\left(g^{\prime} H_{0}\left(H_{T}-H_{0}\right) / H_{T}\right)^{1 / 2}$ and deformation radius $L_{D 2}=c_{2} / f$. The solid lines are the theory for $H=1.5$ and 2 scaled by $\sqrt{g^{\prime} H_{0}}$ and $L_{D}=\sqrt{g^{\prime} H_{0}} / f$. 


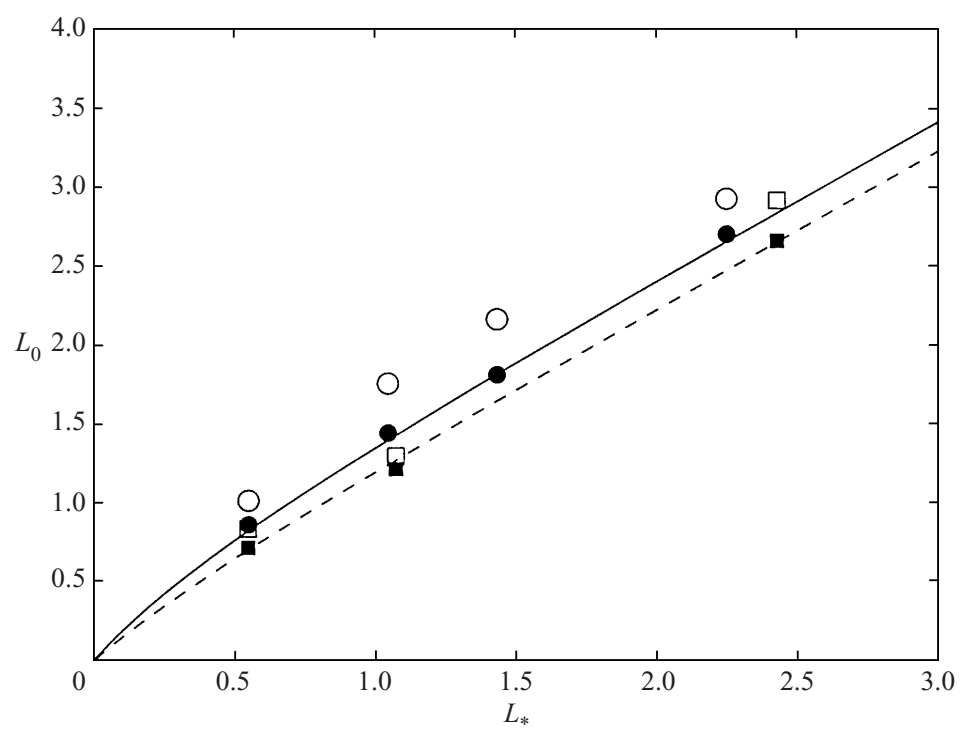

Figure 13. Experimental $L_{0}$ versus $L_{*}$. The open symbols are for the experiments with $H=2$ (circles) and $H=1.5$ (squares). The solid symbols are from the two-layer numerical model run with experimental parameters. Both the experiments and the two-layer numerical results are scaled by the two-layer deformation radius $L_{D 2}=c_{2} / f$. The solid (dashed) line is the single-layer theory for $H=2(H=1.5)$ scaled $L_{D}=\sqrt{g^{\prime} H_{0}} / f$.

$H$ the speeds do not increase consistently with $L_{*}$ as expected. As a test of whether the active lower layer is the reason for the observed discrepancy figure 12 also shows results of two-layer numerical experiments set up to replicate the experiments (e.g. $H_{0} / H_{T}=0.184, g^{\prime}$ and dam geometry). These two-layer calculations give speeds, when scaled with $c_{2}$, which agree qualitatively with the single-layer theory (scaled with $c_{1}$ and $L_{D}$ ), but have generally slower $U_{\text {nose }}$. This implies that the lower-layer effects on the geostrophic adjustment and wave speeds are not the causes of the experimental differences in $U_{\text {nose }}$. Another independent estimate of $U_{\text {nose }}$ is obtained from the observed Kelvin shock speeds $\Gamma_{K}^{o b s}$ (table 1) and Fedorov \& Melville's (1996) weakly nonlinear result (14), which should be reasonable since $U_{\text {nose }} / c_{2}<2$. This indirect estimate gives nose speeds (not shown) which do not reduce the difference between the experiments and theory. However, this is not too troubling because $\Gamma_{K}^{o b s}$ is subject to significant uncertainty in exact arrival time of the shock at the potential vorticity front. The experiments showed clear signs of instability and lateral wave breaking, which were not observed in the two-layer calculations. The absence of instability in the model (which will admit baroclinic instability) suggests that the instability observed in the experiments may be due to non-hydrostatic effects. One possibility is Kelvin-Helmholtz instability of the vertically sheared interface which has been shown to result in similar features in other rotating gravity current experiments (e.g. Griffiths \& Hopfinger 1983). Any resulting mixing could reduce $g^{\prime}$ and reduce the intrusion speed below the adiabatic theory and model results.

The agreement between the observed upstream front width $L_{0}$ and the theoretical and two-layer numerical models is good, though the experimental widths average about $15 \%$ larger than both the single-layer theory and the two-layer numerical results (figure 13). This discrepancy may reflect the effects of the frontal instability and the vertical and horizontal mixing of dye as the fluid slumps under gravity immediately after removal of the dam. 

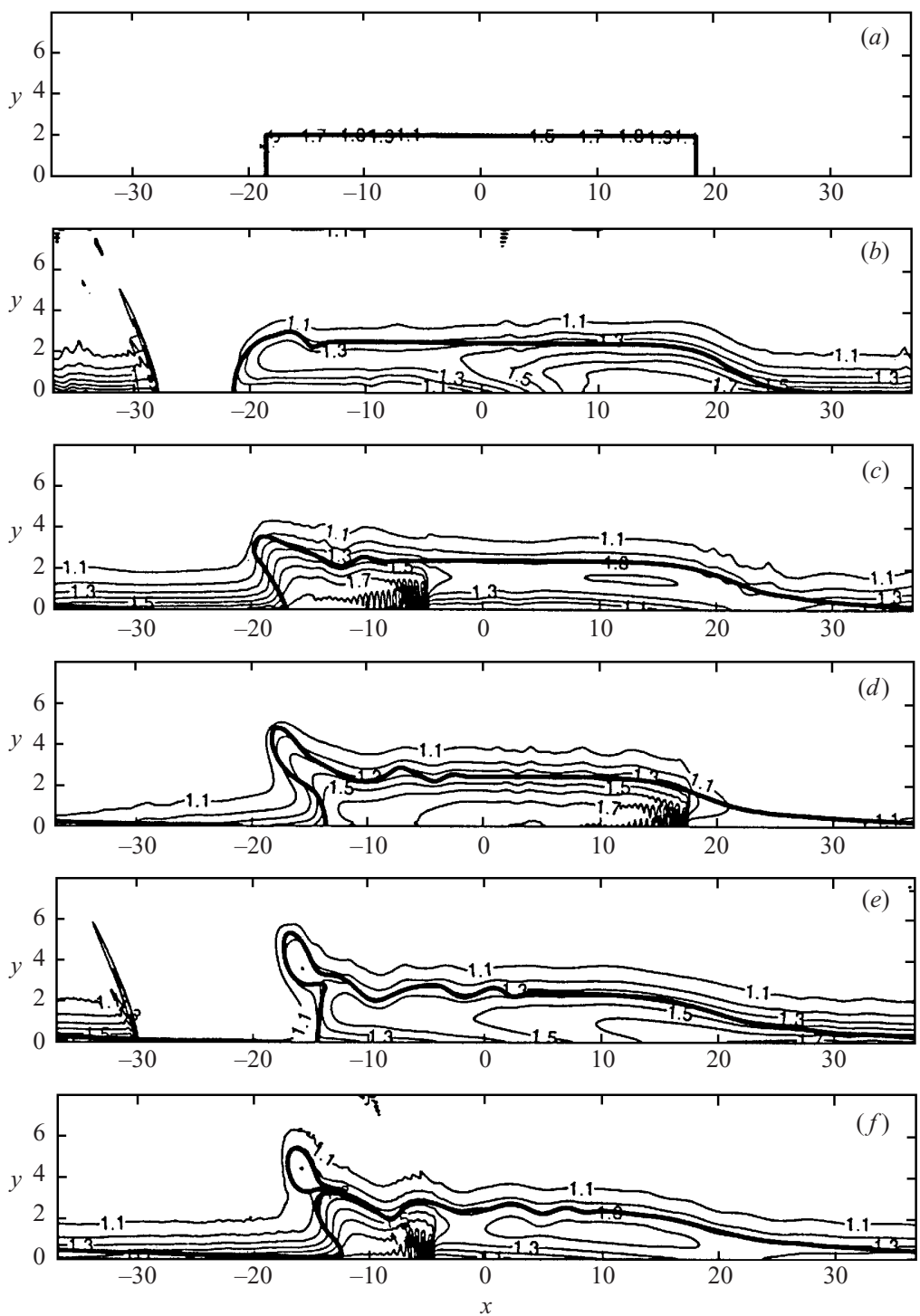

FIGURE 14. Reduced-gravity numerical solution for $H=2$ and $L_{*}=2\left(L_{0}=2.40\right)$ with a finite-length initial condition and periodic boundaries in $x$ to replicate the experimental setup. Contours of the layer depth $h$ (contour interval of 0.1) at (a) $t=0,(b) t=20,(c) t=40,(d) t=60,(e) t=80$, and ( $f) t=100$. The heavy solid line is the $c=0.5$ contour which defines the intrusion boundary. In this run $\Delta x=\Delta y=0.1$.

\section{Further numerical interpretation of the experiments}

The laboratory experiments show that the interaction of the leading Kelvin wave with the trailing end of the low- $q$ fluid results in the offshore ejection of a tongue of fluid and eddy generation. This process was further explored with the reduced-gravity numerical model initialized with a finite-length dammed region and periodic boundary conditions in $x$. Figure 14 shows an example with $H=2$ and $L_{0}=2.40$. The leading Kelvin bore propagates around the domain and collides with the trailing edge of the high-potential-vorticity fluid (panels $b, c$ ). This results in an offshore tongue of 
low- $q$ fluid (panels $c, d$ ) which forms an anticyclonic eddy (panel $e$ ). Surprisingly, the Kelvin bore survives the collision with the low- $q$ front nearly intact and ultimately propagates around the domain to interact with the trailing edge of the low- $q$ fluid a second time (panels $e, f$ ). The bore speed prior to interaction with the low- $q$ fluid (between panels $a$ and $b$ ) is 1.38, while within the low- $q$ fluid (panels $d, e$ ) the bore speed is reduced slightly to 1.12 . The interactions produce wave-like disturbances on the front which intensify and steepen with time. However, the wavelength of these disturbances is generally larger than the frontal waves observed in the laboratory experiments.

The expulsion of a tongue of low-potential-vorticity fluid is a product of the structure of the Kelvin bore. A feature of Kelvin shock is an ageostrophic boundary layer just on the upstream side of the jump (Fedorov \& Melville 1996). This boundary layer is characterized by strong offshore jet $(v=O(u))$ with $x$ and $y$ scales of order the deformation radius $\sqrt{g^{\prime} H_{0}} / f$. Passage of the Kelvin wave through the trailing edge of the potential vorticity front produces a period of rapid offshore advection of the front. The tongue subsequently becomes unstable and pinches off an anticyclonic eddy. The experiments (see figure 10e,f) exhibit vortex pairs, rather than the single anticyclone of figure 14, due to the active lower layer. This process is distinct from the shear layer instability, and subsequent eddy production, triggered by a nonlinear Kelvin wave discussed by Rogerson (1999).

Another interesting feature of the experiments is the stagnation and then reacceleration of the potential vorticity front nose (see figure 11). This is a consequence of the finite length of the dam and the azimuthally periodic structure of the experiments. Upon removal of the dam there is, in addition to the leading Kelvin shock (a wave of elevation, or thickening of the layer), the generation of a Kelvin wave from the upstream end of the dam. This wave is a wave of depression (a rarefaction) and as it propagates downstream it reduces the layer thickness along the wall to nearly the far-field value $(h=1)$. The result is nearly stagnant flow along the wall after the passage of the trailing Kelvin depression. This wave 'turns off' the geostrophic current along the wall, halting the advance of the potential vorticity intrusion. The return of the leading Kelvin shock and trailing geostrophic flow reaccelerates the nose.

This process is illustrated in figure 15 using the reduced-gravity model. The parameters of this run are identical to the previous figure, except that the dam length is $X_{D}=20$ (compared to 37 in figure 14). This change alters the phasing between the leading and trailing Kelvin waves to bring out the process. At $t=20$ (panel $b$ ) the leading Kelvin shock is at $x \approx-32$ and the trailing Kelvin wave is at $x \approx 15$, just behind the intrusion nose at $x \approx 22$. Between $t=40$ and 60 (panels $c$ and $d$ ) the nose is nearly stationary and $u=-\partial h / \partial y \approx 0$ in this region. The Kelvin shock returns (panels $d, e$ ) and the nose reaccelerates. Figure $15(f)$ shows $x_{\text {nose }}(t)$. The time to stagnation of the nose $t_{\text {stag }}$ can be estimated from $U_{\text {nose }}$, the trailing Kelvin depression wave (non-dimensional) speed $c_{d}=\sqrt{H}$ and the length of the dam $X_{D}$, as $t_{\text {stag }} \approx X_{D}\left(c_{d}-U_{\text {nose }}\right)^{-1}$. For the conditions of the example in figure $15, H=2$, $X_{D}=20$ and $U_{\text {nose }}=0.72, t_{\text {stag }} \approx 29$. This is about the time when the nose begins to slow in figure $15(f)$. If the domain were not periodic so that the leading Kelvin wave did not return to reaccelerate the nose, then the leading edge of the potential vorticity front would experience a finite displacement of $\approx U_{\text {nose }} X_{D}\left(c_{d}-U_{\text {nose }}\right)^{-1}$ in the downstream direction. A comparison of $x_{\text {nose }}(t)$ from the two-layer model and the experiments for run 3 is made in figure 11. The qualitative agreement is very good; the quantitative differences are probably due to the slightly slower intrusion and Kelvin wave speeds in the numerical solution. 

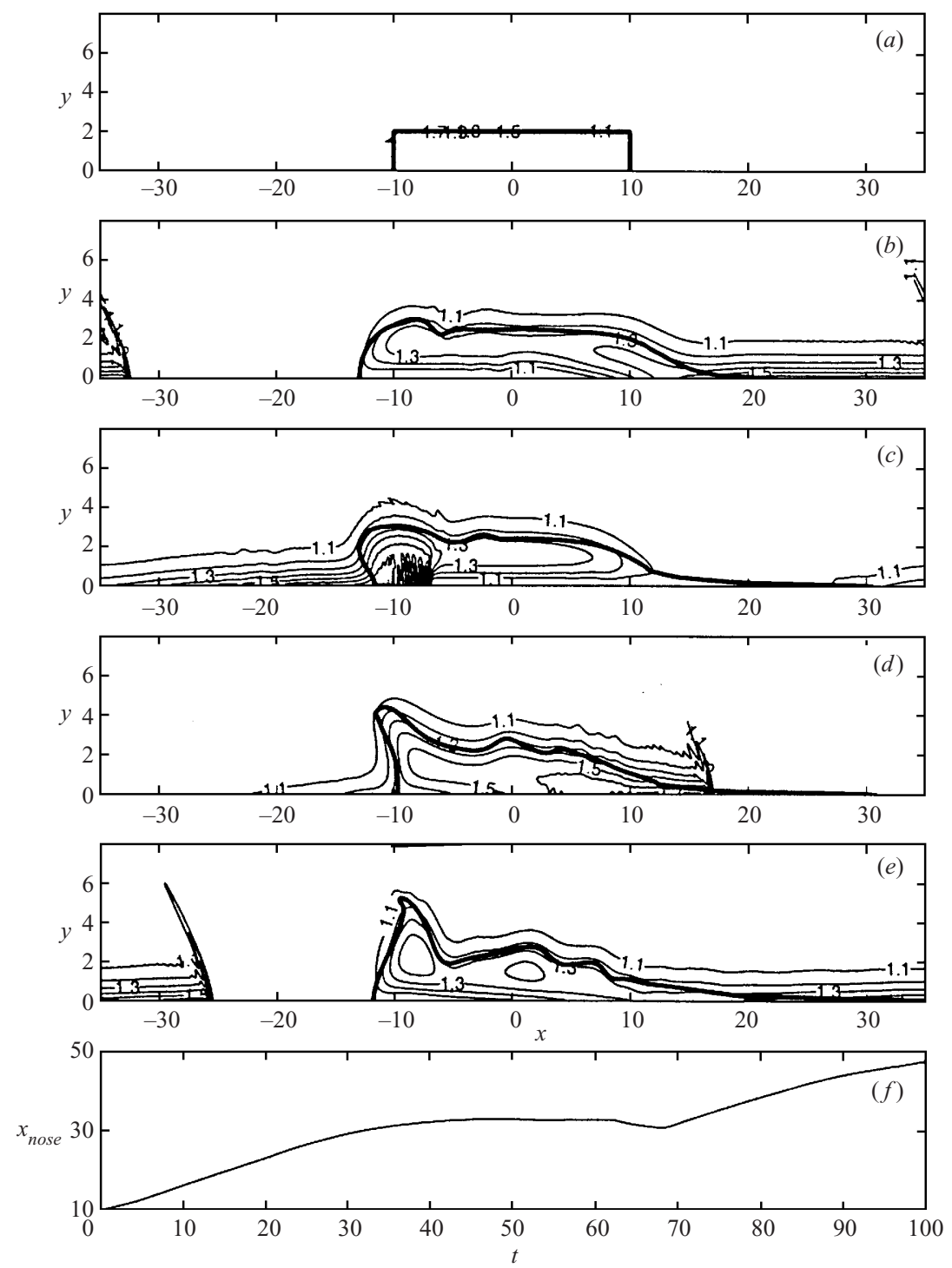

FIGURE 15. Reduced-gravity numerical solution for $H=2$ and $L_{*}=2\left(L_{0}=2.40\right)$ with a finite-length initial condition and periodic boundaries in $x$. The only difference from figure 14 is in the length of the dam. Contours of the layer depth $h$ (contour interval of 0.1 ) at $(a) t=0,(b) t=20$, (c) $t=40$, (d) $t=60$, and $(e) t=80$. The heavy solid line is the $c=0.5$ contour which defines the intrusion boundary. In $(f) x_{\text {nose }}(t)$ is plotted.

\section{Discussion}

We have shown that the modified model (figure 1) of the classical dam-break geometry eliminates some of the theoretical complexities and facilitates understanding of the nonlinear dynamics of boundary intrusions. The main result gives the structure and alongshore propagation of a front separating fluids with different potential vorticity. Oceanic boundary currents typically have widths much smaller than the alongshore spatial scale, so that the alongshore velocity (only) is approximately in geostrophic balance ('semi-geostrophy'). This fact motivated the long-wave theory 
$(\S 2)$, whose main assumptions are verified using the complete shallow-water equations $(\S 3)$.

The qualitative features of this model have been realized in a laboratory experiment $(\S 4)$ but the vertical shear and the small-scale (non-hydrostatic) turbulence are probably more significant here than in the actual stratified ocean or in the numerical experiment, so that the latter is actually more relevant than the lab experiment.

The numerical solution contains many different effects: Poincare waves, Kelvin waves, semi-geostrophic flow, noses of potential vorticity fronts, and collision of the latter with fully developed Kelvin shocks. A virtue of the similarity theory $(\S 2)$ is that it isolates one of these effects by assuming that at some time after removing the dam the fluid adjusts to a slowly varying downstream state (long-wave approximation). The numerical solution justifies this and the lab experiment also confirms that the leading edge of the similarity intrusion is an 'expansion wave' which trails the Kelvin shock. The predicted speed of propagation of the leading edge of the potential vorticity front is in excellent agreement with the numerics (figure 8).

The lab experiment (figure 10) reveals a new and potentially important effect in which a Kelvin shock wave colliding with the rear of a potential vorticity front generates a dipolar eddy in the convergence region. This eddy can then self-propagate away from the boundary, as occurs (figure 14) in the numerical simulation. It is suggested that this may be an effective mechanism for transporting and mixing coastal water masses into the interior ocean.

A rather surprising aspect of our work was the absence of any significant difference between the $1 \frac{1}{2}$ (reduced gravity) and 2-layer calculations (for the conditions used in the laboratory experiment); neither of these models (which are in principle capable of barotropic and baroclinic instabilities) exhibit the frontal wave instabilities seen in the experiments (figure 10). Perhaps the rear end ( $x=-15$ in figure 14) of the front and its downstream propagation prevent the instabilities from forming. In the experiment, on the other hand, these instabilities have already appeared before the rear-end Kelvin effect. Another possibility is that the difference might be due to the aforementioned fact that (unwanted) non-hydrostatic effects appear in the two-layer experiments, but not in the shallow-water equations.

This work is funded by National Science Foundation grants OCE-9726584 \& OCE0092504 (M.E.S.) and OCE-9810599 (K. R.H.). We thank Dr John A. Whitehead for help and discussion of the laboratory experiments. This is Contribution Number 10584 of the Woods Hole Oceanographic Institution.

\section{Appendix}

The coefficients $a_{m n}$ and $b_{m n}(m, n=1-4)$ for the case of finite potential vorticity $(\S 2.4)$ are

$$
\begin{aligned}
& a_{11}=1+H^{1 / 2} \sinh \left(L H^{-1 / 2}\right) \frac{\mathrm{d} A}{\mathrm{~d} U}+H^{1 / 2}\left(\cosh \left(L H^{-1 / 2}\right)-1\right) \frac{\mathrm{d} B}{\mathrm{~d} U}, \\
& a_{12}=\frac{\mathrm{d} A}{\mathrm{~d} U}(H+A), \\
& a_{21}=-H^{-1 / 2} \frac{\mathrm{d} B}{\mathrm{~d} U} \\
& a_{22}=\frac{B}{H} \frac{\mathrm{d} B}{\mathrm{~d} U}+\frac{\mathrm{d} A}{\mathrm{~d} U},
\end{aligned}
$$




$$
\begin{aligned}
b_{11}= & H-1+A \cosh \left(L H^{-1 / 2}\right)+B \sinh \left(L H^{-1 / 2}\right)+H^{1 / 2} \sinh \left(L H^{-1 / 2}\right) \frac{\mathrm{d} A}{\mathrm{~d} L} \\
& +H^{1 / 2}\left(\cosh \left(L H^{-1 / 2}\right)-1\right) \frac{\mathrm{d} B}{\mathrm{~d} L}, \\
b_{12}= & \frac{\mathrm{d} A}{\mathrm{~d} L}(H+A), \\
b_{21}= & -H^{-1 / 2} \frac{\mathrm{d} B}{\mathrm{~d} L} \\
b_{22}= & \frac{B}{H} \frac{\mathrm{d} B}{\mathrm{~d} L}+\frac{\mathrm{d} A}{\mathrm{~d} L}
\end{aligned}
$$

where $A$ and $B$ are given by (33) and (34), and

$$
\begin{aligned}
\mathrm{d} A / \mathrm{d} U & =H^{1 / 2} \sinh \left(L H^{-1 / 2}\right)+\cosh \left(L H^{-1 / 2}\right), \\
\mathrm{d} A / \mathrm{d} L & =U \cosh \left(L H^{-1 / 2}\right)+(1+U-H) H^{-1 / 2} \sinh \left(L H^{-1 / 2}\right), \\
\mathrm{d} B / \mathrm{d} U & =-H^{1 / 2} \cosh \left(L H^{-1 / 2}\right)-\sinh \left(L H^{-1 / 2}\right), \\
\mathrm{d} B / \mathrm{d} L & =-U \sinh \left(L H^{-1 / 2}\right)-(1+U-H) H^{-1 / 2} \cosh \left(L H^{-1 / 2}\right) .
\end{aligned}
$$

The parameters $\alpha, \beta$ and $c$ of (40) are

$$
\begin{aligned}
& \alpha=b_{11} b_{22}-b_{12} b_{21}, \\
& \beta=a_{22} b_{11}+a_{11} b_{22}-b_{12} a_{21}-a_{12} b_{21}, \\
& c=a_{11} a_{22}-a_{12} a_{21} .
\end{aligned}
$$

\section{REFERENCES}

Beardsley, R. C., Dorman, C. E., Friehe, C. A., Rosenfeld, L. K. \& Winant, C. D. 1987 Local atmospheric forcing during the Coastal Ocean Dynamics Experiment 1. A description of the marine boundary layer and atmospheric conditions over a Northern California upwelling region. J. Geophys. Res. 92(C2), 1467-1488.

Dorman, C. E. 1987 Possible role of gravity currents in Northern California's coastal summer wind reversals. J. Geophys. Res. 92(C2), 1497-1506.

Fedorov, A. V. \& Melville, W. K. 1996 Hydraulic jumps at boundaries in rotating fluids. J. Fluid Mech. 324, 55-82.

GiLl, A. E. 1976 Adjustment under gravity in a rotating channel. J. Fluid Mech. 77, 603-621.

Griffiths, R. W. 1986 Gravity currents in rotating systems. Annu. Rev. Fluid Mech. 18, 59-89.

Griffiths, R. W. \& HopfingER, E. J. 1983 Gravity currents moving along a lateral boundary in a rotating frame. J. Fluid Mech. 134, 357-399.

Helfrich, K. R., Kuo, A. C. \& Pratt, L. J. 1999 Nonlinear Rossby adjustment in a channel. J. Fluid Mech. 390, 187-222.

Herman, A. J., Rhines, P. \& Johnson, E. R. 1989 Nonlinear Rossby adjustment in a channel. J. Fluid Mech. 205, 469-502.

LeVeque, R. J. 1997 Wave propagation algorithms for multidimensional hyperbolic conservation laws. J. Comput. Phys. 131, 327-353.

OrLanski, I. 1976 A simple boundary condition for unbounded hyperbolic flows. J. Comput. Phys. 21, 251-269.

PratT, L. J. 1983 On inertial flow over topography. Part 1. Semigeostrophic adjustment to an obstacle. J. Fluid Mech. 131, 195-218.

Rogerson, A. 1999 Transcritical flows in the coastal marine atmospheric boundary layer. J. Atmos. Sci. 56, 2761-2779. 
Stern, M. E. 1980 Geostrophic fronts, bores, breaking and blocking waves. J. Fluid Mech. 99 687-703.

STERn, M. E. 1986 On the amplification of convergences in coastal currents and the formation of 'squirts'. J. Mar. Res. 44, 403-421.

Stern, M. E., Whitehead, J. A. \& Hu, B. L. 1982 The intrusion of the head of a gravity current along the coast of a rotating fluid. J. Fluid Mech. 123, 237-266.

StOKER, J. J. 1957 Water Waves. Interscience.

Tomasson, G. G. \& Melville, W. K. 1992 Geostrophic adjustment in a channel: nonlinear and dispersive effects. J. Fluid Mech. 241, 23-58.

Whitehead, J. A., Leetma, A. \& Knox, R. A. 1974 Rotating hydraulics of strait and sill flows. Geophys. Fluid Dyn. 6, 101-125. 